\title{
Towards a paradigmatic description of context: systems, metafunctions, and semantics
}

Ruqaiya Hasan

Correspondence:

Ruqaiya.hasan@gmail.com Department of Linguistics,

Macquarie University, New South

Wales 2109, Australia

\begin{abstract}
This article hopes to make a contribution to the growing body of work on the paradigmatic description of context of situation. Having considered the limited achievements of earlier work on context, I offer a fairly detailed account of what is entailed in producing a consistent and precise description of a linguistic category by using the principles and methods of the paradigmatic mode of description devised by the systemic functional linguistics. The system network is a powerful device for an accurate and orderly representation of such a description. The paper will close with remarks on the importance of a scientific analysis of language use (parole) in developing a comprehensive post-Saussurean linguistic theory.

Keywords: Context-metafunction resonance; System networks (sys-net); Individual system; Siultaneous sets; Options; Choice; Entry point; Realisation; Selection expression (SE); Accuracy; Consistency
\end{abstract}

\section{Background}

The main aim of this paper is to contribute to the description of the contextual parameters of field, tenor and mode by focussing on what part each parameter plays in the production of a text, which, in fact, is nothing more than the meaning-wording produced by the interactants as they speak with reference to some social practice. Given that these parameters were first introduced nearly half a century ago (Halliday et al., 1964), and that they have been in use over this long period, one might wonder why the issue has arisen now. The significant factor is the move in the last couple of decades to produce paradigmatic accounts of field, tenor and mode; and these accounts are now becoming more widely available (Bowcher 2007; 2014; Butt 2004 mimeo; Hasan 1999; 2009a; 2013; 2014; in press a). There are suggestions for the 'unification' of options from distinct system networks, and some evidence of disagreement as to the task that may be assigned to each of the three parameters. I hope to contribute to these debates (i) by presenting a brief history of the description of context from the inception of the idea to date; (ii) by exploring the bases of accuracy and consistency in paradigmatic linguistic descriptions represented by the use of the precise conventions of the system networks; and (iii) by pointing to the significant part played by the description of context, carried out in keeping with the architecture of the Systemic Functional Linguistics (SFL): I see this model as a post-Saussurean theory whose aim is to provide a scientific account of both the system (langue) and the use of language (parole) ${ }^{\mathrm{a}}$. 
To this end, the next section on register variation and context of culture and situation will provide relevant background information about the contextual parameters. The focus here will be on certain problems in the identification of the contextual features which finally provided the impetus for the application of the paradigmatic description to their study: I will argue that the roots of these problems go far back into the history of contextual description. The account will begin by relating the concept of context to that of register; it was the classification of register that led to the introduction of the three parameters of field, tenor and mode. Some problems in conceptualising their role will be brought to attention together with steps to specifically address those issues. In the following sections, the formal conventions and the semantics of the language of paradigmatic description will be discussed. Some important questions here are: how the domain of description is identified and how its integrity is maintained throughout the representation of its description presented as system networks; how the relevance and the validity of systemic options may be established. Representing description in the form of system networks enforces order: the method is designed to create explicit and non-ambiguous relations across the options. Their validation is tested by their realisational relations to language, which guards against contradictions within the description. This constitutes the heart of my argument: it presents my understanding of what doing paradigmatic description means in practice. Implicit in those statements are the reservations against some of the actual systemic contextual representations as well as the proposals for future directions. By way of concluding remarks: the value of a scientific study of context closes the discussion by explicitly indicating the role of a scientific description of context in the ecology of SFL theory. Treating that theory as a post-Saussurean version of Saussure's linguistics of langue', I present arguments for the inclusion of parole: I will argue that variation cannot be ignored because even within a synchronic état de langue it could affect the 'system', i.e., langue; and without the support of a competent scientific theory of parole neither the fallout from linguistic variation can be adequately handled, nor can Saussure's 'associative bonds' receive the validation they require to fulfil the role he assigned them as the most important methodological instrument in his 'morphology of meaning'. In fact the method for studying Saussure's 'associative bonds' is best developed in SFL in the shape of paradigmatic linguistic description, as section The paradigmatic description of contextual parameters demonstrates. Apart from this, there is a significant hypothesis in the systemic functional model, namely that, there is a systematic prehension between the linguistic metafunctions and the internal organisation of natural language, the basis of which lies in how speakers use language as needed in the performance of social practices.

\section{Register variation and context of culture and situation}

The first linguist to appreciate the rich implications of Malinowski's context of situation (1923; 1935) was Firth (1957; 1968), though he was also quick to note its 'instance based' view of 'situation' as simply 'events considered in rebus' (1957: 182). Halliday appreciated not only Firth's insights into the relations of context and language, but also his efforts to transform context from 'a bit of the social process' to a viable 'schematic construct' suitable for applying to the analysis of 'language events' (Firth, 1957: 182). This understanding was injected into SFL by Halliday, who positioned 'text' as language event, relating the variations of text in context to inherent linguistic phenomenon.

One cannot help but notice with interest that today it is Malinowski's concept of context, perhaps strengthened as needed with ideas from Halliday's SFL, that supports 
the drive towards the multimodal 'text' analyses highly popular. Halliday's method for bringing order to the chaos of situation was to focus on the 'speech event' without either ignoring or privileging the extra-linguistic information. SFL is widely viewed today as friendly to text analysis, especially where the concern is with linguistic meaning-wording. In contrast to these two frames, Firth's account falls in the middle: it rejects Malinowski's 'instance-based' 'holistic' view of the context of situation, and it fails to foreground the specificity of the linguistic analysis of 'speech events'. Not many practitioners of SFL use Firth's inspiringly rich but sprawling and sometimes contradictory framework for the contextual analysis of meaning either as instance or as system ${ }^{\mathrm{b}}$. Often, even linguists announcing a Firthian orientation, will 'elaborate' Firth's ideas typically in terms of Halliday's methods for register analysis.

The commitment of SFL to language as the central object of enquiry is evident in the claim that the goal of linguistics is to provide 'a theory of how language works' (Halliday, 1961: 241); and later (Halliday, et al. 1964: 5); To study language scientifically means to construct a ... theory of how language works, and to derive from it certain exact methods for describing language.' It is obvious that language works in time and space; but the essence of 'situation' as in 'context of situation' does not lie in the situation's spatio-temporal dimensions per se: what imbues it with relevance for the linguist is the function of talk in the performance of social practices. Entry in the world of extra-linguistic phenomena has value for the linguist in as much as it helps explain the 'how' and 'why' of language: after all, that is where the data of experience will be found. The issue is not a choice between 'inside' and 'outside'; rather, it is the question of maintaining a balance in their relations while keeping the goal of the theory in view. The analysis of 'discourse' acts as the measure of the analyst's success in maintaining this balance.

\section{Register as a linguistic variety and text as instance}

Responding to this focus on language, the category of 'register' emerged from a concern with the inherently variable nature of language. According to Halliday et al. (1964) this inherent variation in language is best viewed in relation to language use and language user:

[there are, $\mathrm{RH}$ ] varieties according to users (that is, varieties in the sense that each speaker uses one variety and uses it all the time) and varieties according to use (that is, in the sense that each speaker has a range of varieties and chooses between them at different times). The variety according to user is a DIALECT; the variety according to use is a REGISTER. (Halliday et al., 1964:77).

The concept of register as a 'variety according to use' was developed further (e.g., 1964: 87-89):

Language varies as its function varies; it differs in different situations. The name given to a variety of a language distinguished according to use is 'register'... It is only by reference to the various situations, and situation types, in which language is used that we can understand its functioning and its differences. Language is not realized in the abstract: it is realized as the activity of people in situations, as linguistic events which are manifested in a particular dialect or register.' 
As their text shows, despite the 'or' above, the authors thought of the two varieties as cutting across each other: a speaker positioned in a specific context of situation would in all likelihood speak with relevance to it; in other words, he would speak 'in' register. At the same time, speaking has also to be done 'in' dialect, whether standard or not is immaterial from the perspective of linguistic variation. Besides this, each act of speaking displays also some indication of the syndrome of linguistic features unique to an individual speaker's ways of speaking: every feature in the syndrome is part of the system; what belongs uniquely to the speaker is its formation as a particular syndrome. Linguists recognise this category as 'idiolect', though sometimes today its description is presented as the study of a speaker's identity. By definition, idiolects cannot be recognised in any segment of the community as a linguistic variety (Hasan, 1973).

Each of these categories represents a distinct aspect of the naturally occurring text: each text indicates some register, some dialect, and idiolectal syndrome ${ }^{\mathrm{c}}$. In the early 1960's, the term 'text' had been much the same in Halliday's writing as in the other models of general linguistics. To quote, 'The data to be accounted for are observed language events, observed as spoken or as codified in writing, any corpus of which, when used as material for linguistic description, is a "text".' (Halliday 1961: 243; emphasis introduced). 'Text' now represents a qualitatively different, more mature, concept though retaining the same name: today in SFL, we see 'text' as naturally occurring language use, therefore having a social function, and possessing the attributes of texture and structure (Halliday and Hasan, 1976; 1985; Hasan, 1978); as an instance of register, the text may be simple or complex (Hasan, 1999); and, most importantly, in instantiating the linguistic system, this is where every form of linguistic regularity as also every move in innovation will manifest itself (Halliday, 1991/2007b) . The $^{\text {T }}$ text, thus, represents a measure of what language is able to do: it is a reliable source of insight into the power of the language system (Matthiessen, 2009).

\section{Contextual parameters and the classification of register}

The genesis of contextual parameters too lies in register variation. They were assigned the task of furnishing linguistic evidence for classifying registers (Halliday et al. 1964: 90; original emphasis):

There is enough evidence for us to be able to recognize the major situation types to which formally distinct registers correspond; others can be predicted and defined from outside language. A number of different lines of demarcation have been suggested for this purpose. It seems most useful to introduce a classification along three dimensions, each representing an aspect of the situation in which language operates and the part played by language in them. Registers, in this view, may be distinguished according to field of discourse, mode of discourse and style of discourse...

From the ordinary speaker's perspective, register is perhaps as remote a concept as language system: in everyday life we encounter neither system nor register but text. Concepts such as language system, register, register variety (I believe 'register variety' was renamed as 'text type' by Halliday 1991/2007b) are abstractions. Text is where the journey towards system begins; and the concept of text is pivotal to validating the categories of register.

Following the above extract are 'introductory remarks' on each parameter; a selection from each is presented below, beginning with 'field of discourse' (Halliday et al., 1964: 90): 
'Field of discourse' refers to what is going on: to the area of the operation of the language activity. Under this heading, registers are classified according to the nature of the whole event of which language activity forms a part. In the type of the situation in which the language activity accounts for practically the whole of the relevant activity, such as an essay, a discussion, or an academic seminar, the field of discourse is the subject matter. On this dimension of classification we can recognize registers such as politics and personal relations, and technical registers like biology and mathematics.'

'Mode of discourse' is the next parameter to be discussed (Halliday et al., 1964: 91):

'... this [i.e.,' mode of discourse', $R H$ ] refers to the medium or mode of the language activity, and it is this that determines, or rather correlates with, the role played by the language activity in the situation. The primary distinction on this dimension is that into spoken and written language, the two having, by and large, different situational roles...'

Last mentioned was the parameter called then the 'style of discourse' (Halliday et al., 92-93):

Third and last of the dimensions of register classification is 'style of discourse', which refers to the relations among the participants. To the extent that these affect and determine features of language, they suggest a primary distinction into colloquial and polite ('formal', which is sometimes used for the latter, is here avoided because of its technical sense in description). This dimension is unlikely ever to yield clearly defined, discrete registers.

The meaning of the word 'style' varies a good deal, especially in the field of literary criticism. It was Gregory (1967) who offered 'tenor of discourse' as a replacement for this 'undesirable' term, and the label has prevailed. Halliday et al.'s three parameters resemble Firth's 'categories' for use in 'linguistic work' (1957: 182); the differences between the two frames are as significant as their similarities (Hasan 1995). I close this discussion with a remark by the authors (1964: 93):

It is as the product of these three dimensions of classification that we can best define and identify register. ... The formal properties of any given language event will be those associated with the intersection of the appropriate field, mode and style.

\section{Parameters evolving: major developments and major problems}

Like most newly introduced theoretical categories, the contextual parameters as seen in relation to their linguistic correlates have also undergone changes and developments. A viable and verifiable view of their correlations is essential in view of the status of register as 'linguistic variation according to use'. Below is presented a brief history of the developments made in that pursuit. Pushed to its limits, probably each development would have moved SFL towards a viable method for establishing correlation between the text's language and its context. As it happens, each to date has ended in what might be described with a touch of wry humour as 'snatching defeat from the jaws of victory'. As I commented in introducing this paper, the current problems have become more visible with attempts to describe context paradigmatically, but their genesis lies in the earlier studies of context. In the following sections, I present my views on this earlier history. 


\section{Developments and outcomes in text-context studies: (1) early FTM}

The first development in this pursuit was the hypotheses as presented above. As Halliday et al. (1964: 90) acknowledge, other lines of demarcation for context of situation had been introduced, the most important being Firth's (Butt, 2001; Hasan, 1995). However, the parameters of discourse presented by Halliday et al. (1964: 89-94) do differ significantly from the earlier discussions: here, for the first time, each parameter was conceptualised as facing both in the direction of the context of situation, representing 'an aspect of the situation in which language is operating' and also in the direction of the text, by representing 'an aspect of the part played by language'. I see this as an early form of using realisation as a means of bringing context and text together.

The requirement of correlation between linguistic and situational features imposed a grid on both context and text by using that theoretical concept of 'realisation': without this relation register classification could not get off the ground. Once the fact of correlation is accepted, all that the analyst need to do is to find some reliable recognition criteria either for the features relevant to each contextual parameter or for its linguistic correlates; either identified correctly would have led to the other. In fact, things did not work out this way. Below I suggest an explanation why, half a century later, SFL has still not established viable principles for the recognition of either the situational or the linguistic features, thus leaving register classification in limbo.

Eirian Davies (2014) has commented on the paucity of viable information on registers prior to the research at University College London in the second half of the 1960s. The Hallidayan conception of register required a fairly precise understanding of both situational and linguistic facts. But in the words of Bernstein, the 'language of description' had not developed as yet to enable an adequate conceptualisation of either of the two unknowns. The words that named the important concepts were new arrivals in a nascent theory. It is not that words such as 'situation' 'field' and 'mode' were too exotic; their meaning in 'ordinary language' hardly seems problematic. But as Firth had warned, ordinary words, when used to construe 'schematic constructs', tend to acquire a somewhat different value and identity. They begin to act just like words that name 'grammatical categories' such as, the 'simple present' which is neither 'simple' nor necessarily 'present'. Similarly, the terms being used to identify linguistic features proved not quite up to the task.

The term I as a novice found most problematic in context studies was 'activity' (Halliday et al. 1964: 90): in ordinary life, the word has many meanings and each seems clear in its 'context', but what exactly did it mean in the description of field of discourse? Here it seemed to have multiple values: it was not clear if the word referred to precisely the same phenomenon in its various appearances, such as 'social activity', 'relevant activity', 'language activity'. There were also 'descriptive references' such as 'what is going on', and 'the area of the operation of the language activity'. Sometimes 'the whole activity' was said to consist of two kinds of 'activities', a 'language activity' which 'assisted' 'the whole event', in which case it would seem that the 'whole event' was to consist of both 'language activity' and some other kind of 'activity' which was not linguistic. At other times, the 'whole of the relevant activity' could be accounted for 'practically' by 'language activity' (did that mean language was fully constitutive of such a 'relevant activity', so that nothing else was needed to recognise what was going on?). Equally problematic was the word 'subject matter', a term that has continued to be used in a sense quite close to 'the content, specific to some specialised domain'; but the whole of the field could clearly not 
consist of subject matter. If something is going on, then some action seems to be required somewhere; and in the nature of things, the domain of activity is defined by the classification of goings on. Where did 'subject matter' fit?

The problem of the meaning of 'activity' in field was not made any easier by the account associated with the mode of discourse: this was characterised as 'refer[ring] to the medium or mode of the language activity'; 'it (i.e., mode) ... correlates with the role played by the language activity in the situation' (1964: 91). One possible interpretation of mode is that it was a means of content relay, obviously needed in 'concerted action'; but 'phonic' is not identical in meaning to 'spoken' as in 'spoken style', nor does the spoken style become 'graphic' when written down. How did the role of language clarify this situation? As for 'language activity', was it part of field, or of mode? What would be the 'language activity' in field, when 'interviewing' is the 'role of language'?

To my mind, the entanglement of field and mode is not new; it dates back to this early stage. By contrast, tenor, a more 'exotic' term, escaped this fate, though it too was distorted in other ways (Hasan in press a); it began to be treated like a thing apart from field and mode. And yet, features of style/tenor do clearly correlate with interactant relations, as well as the kind of linguistic activity, and/or mode. In early SFL, some evidence of discussion about the scope of the parameters among interested scholars can certainly be found, but solutions usually took the form of suggesting a greater number of parameters (for example, Fawcett, 1980; Gregory and Carroll, 1978; Ure and Ellis, 1977; Hasan, 1973). And those suggestions appear not to have had much impact except locally; the uptake in the SFL community suggests the original three parameters are more widely used - but what the different users mean by them is not necessarily the same thing.

Could one not have used language patterns as the first step in the search for correlates? When the parameters were first introduced, the register specific linguistic patterns were stated in terms of grammatical and lexical patterns, including a selection of idiomatic expressions 'strongly marked as register specific', e.g., 'how are we today?' as indicating 'a doctor-patient style'; and 'denture' as part of the language of advertisement. Hasan (1973) drew attention to the serious drawbacks in this itemising approach restricted to 'wording' alone ${ }^{\mathrm{e}}$. But by this time, Halliday had moved to a more abstract means of classifying linguistic form. This was an outcome of the paradigmatically oriented description of lexicogrammar. Specifically, when the system networks pertaining to the various linguistic patterns were examined, this revealed a grouping of options organised fairly systematically with reference to generally recognisable semantic domains, i.e., linguistic meaning. This led to the recognition of 'metafunctions' pertaining to language, which soon became a hallmark of SFL (Halliday, 1967-68; $1969 ; 1970 a ; 1970 b ; 1973 ; 1979)$. It revolutionised the SFL view of language, with significant consequences for the realisation of contextual features. That story merges with the second development in language-context relations.

\section{Developments and outcomes in text-context studies: (2) CMR hypothesis}

The metafunctional perspective suggested that the form of language has evolved along functional lines, with different systems of content being oriented to different linguistic metafunctions. With this metafunctional orientation, system networks became instruments for revealing the organisation of the meaning potential of language, leading to the second major development in the exposition of the relations of text and context. 
SFL recognises four metafunctions: (i) The 'experiential' concerns the resources for the exchange of experience: the lexicogrammar of languages encodes human experience of the external and internal world as processes of various kinds occurring in time and entailing entities as participants, as well as specifying circumstances that elaborate aspects of process-participant configurations. (ii) The 'logical' metafunction concerns the relations between the various individual process-participant configurations; it also elaborates on the nature of entities, processes and circumstances by relating them in different ways to a set of properties. According to Halliday (1973), these two metafunctions are closely related and together they have been referred to as 'ideational'. (iii) The 'interpersonal' metafunction enacts social relations, including the speaker's assessments of possibilities, the evaluation of phenomena, attitude to self and others as well as commitment to the interactive process. Finally (iv) the textual metafunction contributes to 'the creation of text' (Halliday, 1973: 99): the meaning wording patterns oriented to the textual metafunction encode 'the structure of information, and the relation of each part of the discourse to the whole and to the setting' (Halliday, ibid.). Unlike other functional theories, in SFL, the metafunctions work simultaneously and no metafunction is more powerful, more authentic than the others; there is no hierarchic order. According to Halliday (1973: 100):

With only minor exceptions, whatever the speaker is doing with language he will draw on all three components of grammar. He will need to make some reference to the categories of his own experience - in other words, the language will be about something. He will need to take up some position in the speech situation; at the very least he will specify his own communication role and (will) set up expectations for that of the hearer - in terms of statements, questions, response and the like. And what he says will be structured as 'text' - that is to say, it will be operational in the given context.

Halliday has often suggested that metafunctions resonate systematically with the three contextual parameters, which are realised as the relevant meaning-wording that is equal to producing the texts. Thus (i) the linguistic patterns oriented to the experiential metafunction would typically correlate with the features in the system of field; (ii) those deriving from the interpersonal metafunction would typically correlate with the features in the tenor of discourse, and (iii) the textural and organisational resources of the textual metafunction would typically correlate with the mode of discourse. In short, the 'default' linguistic realisation of the features pertaining to the three parameters are predicted by reference to the metafunctional orientation of the lexicogrammar and semantics: the formulation leaves open the possibility of departures from the highly probable ${ }^{g}$. This probabilistic formulation of the context-metafunction resonance (CMR) was greeted with a lively controversy (for some detail, Hasan, 1995). Those expressing objections never took any notice of the fact that in the study of sociolinguistic variation, predictions about linguistic features correlating with situational ones such as age, gender, geographical and/or social provenance, have typically been stated in probabilistic terms, and continue to do so even today.

When in 1964 Halliday et al. had introduced the parameters, presenting a perspective on both the nature of the context of situation and of the linguistic features correlating with the three parameters, this, despite its shortcomings, had been a considerable improvement on the previous rather gross statements about the relations of situation and language. The context-metafunction resonance (CMR) hypothesis was a huge step forward. First, it had 
the potential for providing more specific guidance for recognizing the formal linguistic features capable of acting as the typical correlates of the features pertaining to each situational parameter; rather than citing items, it became possible to identify them as patterns of transitivity, mood, aspects of modality, modulation, or the categories of cohesion. Second, the postulate of the specific contextual parameters raises a pertinent question about the proposed number of parameters as well as the linguistic metafunctions: why this number of parameters/metafunctions, and not any other? The CMR hypothesis offered the possibility of treating the nature of human interaction as the origin of both, the parameters and the metafunctions, resolving both issues with one stroke. According to Halliday (1973) the proposal had been based on a pilot research; that, for him, was reason enough for a cautious probabilistic formulation of the hypothesis. It naturally called for searching ways of checking on its validity. Surprisingly, instead of debating the original probabilistic hypothesis, the view among the SFL scholars was that the hypothesis had failed to provide a 'context metafunction hook up device' that could hang the three specific metafunctions, one on each of the three contextual pegs, thus resulting in making absolute statements, not probabilistic ones. The more relevant need had been to search for ways of testing the validity of the probabilistic CMR hypothesis, i.e., the one that the authors had actually formulated $^{\mathrm{h}}$. In the event, no aspect of the hypothesis received careful scrutiny: the outcome was to follow the same rather intuitive style of contextual description which had been in vogue since Halliday et al. (1964). The problem of register classification has naturally remained unresolved: lacking serious enquiries, the conceptual visibility of register has been jeopardised.

\section{Developments and outcomes in text-context studies: (3) GSP hypothesis}

The third move was a conscious attempt to provide a viable criterion for register classification. The approach required treating each parameter as a resource that offered choices from a large set of contextual options: the total set of options chosen from the three parameters would together count as the contextual configuration (CC) of the text. The CC would represent an occasion of talk (aka context of situation) as a particular instance of some situation type (for discussion, see Halliday and Hasan, 1985).

Hasan (1978) was an attempt to demonstrate that a specifiable class of choices in a CC motivates the over-all structural shape of the text type. Predictive claims of this kind are based on an examination of the data of language use, whose systematic analysis must focus on the shared dis/similarities across the many events. The distinction between a text and a text type becomes critical. Every naturally occurring text is an individual; it will display some features specific to it, even if just those correlating with its material situational setting (Hasan, 1985). The study of a unique occurrence has, no doubt, a value. But so far as the study of register variation is concerned, this is less significant than those properties shared by a number of instances: the latter alone can lead to viable statements of correlation between texts and contexts. This was the perspective adopted in the study of correlation between textual structure and the features of the CC.

The 'over-all structural shape' presented in Hasan (1978) is better treated as a generalised formula. It consists of a configuration of functional elements whose mutual relations are calibrated in such a way as to allow its use to describe the structure of not only a specific text type but also a range of other related text types. Each member of that range of text types will have some structural properties in common with other members: no individual text type will have the same structural shape as any other, and 
none will be entirely different. The entire range of such text types will constitute a single register family. The variations across text types are clearly not accidental. They are based on the text-context relations: underlying these similarities and differences in the structural shapes of the members of a register family there will be specifiable similarities and differences in the selection of features from the dimension of field and/ or tenor and/or mode of discourse. The actual structural shape of a text is not the achievement of that particular text: despite its uniqueness, it is recognised by its regularities which arise from the speaker's meaning-wording as a response to the context of situation: the text is realising in a specific type of CC. The study of register is a study of the regularities between the features of $\mathrm{CC}$ and their realisation as text.

This generalised structural formula eventually got labelled as the 'Generalized Structure Potential' (GSP) ${ }^{\mathrm{i}}$. And from the above perspective, the GSP is clearly relevant to register classification. I would go so far as to claim that it is, in some ways, analogous to the system network. For example, a system network treats its point of origin as its ultimate 'descriptum', i.e., its object of enquiry. And in the course of this enquiry, it creates a number of 'selection expressions' each of which represents the properties of one specific sub-category of the descriptum; these sub-categories are closely related to each other in explicitly specifiable ways. The GSP resembles the system network in these respects: it too describes the structure of a specific register family. In the course of doing this, it produces a range of 'derived structures' each of which pertains to one and only one register variety (aka text type). It is these various text types that together represent one specific register family identified by the GSP. This allows a specific derivation of structural shape as pertaining to one particular register variety; and the register varieties, systematically related to each other, represent a register family. The system network creates many visibly laid out paths each of which can be represented as a 'selection expression', each specifying one sub-category of the ultimate descriptum. The GSP does not produce visibly laid out choice paths, but it explicitly signals those features whose selection would be the realisation of some systematic variation across the derived structures: the derived structures do not vary accidentally; they vary with predictable perturbations in the configuration of the underlying context. In all, the GSP visibly indicates three classes of phenomena:

- the status of its functional elements is visibly indicated: an element is either obligatory, i.e., it defines the nature of that register family, or it is optional, i.e., the appearance of the elements is subject to specifiable variation in the underlying CC;

- the order in sequence of the functional elements that can relate to each other is visibly indicated, some being fixed vis a vis others, and some free within specifiable limits;

- the possibility of recursion/reiteration for some element is visibly indicated.

Just as no systemic choice path will ever describe an ill-formed category of language, so also no GSP will accommodate twists and turns that are 'accidental'. This is for a good reason: in general, systems are known by their regularities; they are not known by their accidental variations. The accidental textual moves are not very likely to realise any part of the $\mathrm{CC}$ that would be recognised as necessarily relevant to any social practice underlying a register. 
This third development had been directly focussed on register classification, the quest for which had led to the postulate of the three contextual parameters. It invited research on closely related fronts, namely: (a) the derived structure pertaining to register varieties; (b) the $\mathrm{CC}$ features underlying its functional elements; (c) the environmental conditions for the operation of the obligatory and optional elements; and (d) their modes of realisation by semantic categories which are themselves realized as lexicogrammatical patterns. The payback from the uniqueness of each GSP and its battery of derived structures would have been significant for attempting register classification both at lower and higher degrees of delicacy: being unique, particular derived structures could have been tested as recognition criterion for the specific register variety, and by implication, also for the related register family. Underlying the obligatory elements of each derived structure of a GSP is a set of the CC features (a 'syndrome'), and realisational pairing with meaning-wording is a critical means of testing the 1973 CMR hypothesis. So it had seemed that attention to issues raised by the GSP analysis of texts could have been valuable for testing CMR hypothesis. However, the preoccupation with mistaken ideas of 'science', and the attraction of the 'dynamic' were then the focus of attention. Register classification remained an obscure problem. The SFL research on both context and register has suffered in consequence.

\section{Developments and outcomes in text-context relations: (4) ARC hypothesis}

The fourth relevant move came some eight years later in exploring Vygotsky's concept of 'semiotic mediation' (Vygotsky, 1978). The questions were: how is culture mediated to the neonate? when does the ontogenesis of semiotic, as opposed to linguistic, mediation begin, and how? Halliday's account of protolinguistic communication between the infant and the care-givers (Halliday, 1973; 1975; 1994) had already highlighted the central role of context in the exchange of meaning. Soon scholarly research was to focus on the communication of only weeks old neonates with their care-givers (Brazelton et al., 1974; Trevarthen, 1974; Lock, 1978). The concept of meaning exchange had to be reinterpreted in this context: semantic phenomena, i.e., mediation by means of linguistic meanings was obviously out of question at this stage. Even those linguistic items uttered by the adult care-giver could hardly be taken as conveying the semantic value of the utterance to the infant. What was it that was being communicated by the communication, and how? Halliday had suggested it was an exchange of personal affect and attention - a classic 'inception of reciprocity' in terms of Brazelton et al. (1974). The research led to the conclusion that the ontogenesis of 'semiotic mediation' could not begin with language - what is needed is an embodied affordance for producing/recognising 'meaning, or significance', perhaps independent of the conventional semiological systems, a kind of direct move from sensation to the internalisation of 'meaning' as significance and relevance - we just do not have the word for that 'meaning-like-thing-which-is-not-quite-like-linguistic-meaning-yet'. This obviously raises the question: is this communication without any context? If not, then how to outline the frame for the contexts pertaining to neonate communication which, as Brazelton et al. emphasise, can and do vary significantly within and across communities, if only because the adults are 'acculturated'.

So the search was for a contextual frame that could be applied to a social practice of any kind where cultural mediation was not limited only to the linguistic means or 
heavily dependent on 'social conventions'? Here it is relevant that social practice covers a much wider domain of human experiences than does register, simply because the latter consists of language entrained apropos some social practice. So, the 'context of discourse', specific to register, could not be taken as a viable frame for social practices of all and any kind for all normal human beings. On the basis of an informal survey of a variety of social practices, it appeared very probable that the undertaking of any must entrain three domains of human experience, namely, Action, Relation and Contact (ARC) (Hasan, 2001:6). A social practice entails some action (activity/doing/act), significant enough to attract the co-engagement ${ }^{\mathrm{k}}$. Being social, some relation is implied between the doers even if just that arising from doing something together (c.f., 'agentive role', and 'social distance', Hasan 1978): in the nature of things each participant is socially positioned (Bernstein 1990); they cannot but be 'related'. And there has to be some way of establishing contact between the actors of those actions, i.e., some means of enabling access to what is going on; without this, both the initiation and the pursuit of the shared activity would be jeopardised. The concept of ARC is relevant to this discussion since it can be used as a template specifying the triadic 'situational' support that every social practice requires. Using language to perform an action with an 'other' is clearly a variety of such activity: the dimensions of the context of situation, Field, Tenor and Mode (FTM), pertaining only to linguistic social practices, can therefore be viewed as a specific case of the ARC template. This mode of validating contextual parameters by reference to ARC has significant implications.

First, taking FTM as a more specific instance of ARC provides a clearer guide to the concerns of each contextual parameter: there is a basis in the ARC frame for identifying certain features of the context of situation as belonging to one parameter rather than another. For example, if field is taken as pertaining to Action, then sphere clearly belongs to field; mode as Contact is a way of facilitating communication, materially, i.e., by embodied means and/or 'virtually' by semantic means; and tenor as Relation concerns speaker/addressee as socially positioned, culturally specific co-actors in an activity that requires linguistic acts either with or without the physical ones.

Second, and following from the above, this conceptualisation of FTM should provide a comprehensive account of the situational features relevant to the exchange of the meaning-wording by the speaker-addressee: therefore, logically, it should include the valid features of other variant models such as Hymes' SPEAKING (1986: 59-65) or Gregory's 'functional tenor'. The latter for example is implicit in the choices from type of action and sphere of action from the system of field; thus, if the action is consultation with a doctor, you could hardly have a 'functional tenor' such as exchanging goods for money.

Third, seen in this perspective, the nature of the parameter is very likely to validate the 1973 CMR hypothesis: the metaphor of 'resonance' is apt for referring to the reciprocal relations of context and metafunction. The metafunctions have evolved in language being used as a form of action, as a means of enacting interpersonal relations, and also as a means of creating relevance, continuity and coherence in interaction. These resources are common to all languages. If field is far more likely to be realised by the meaning-wording resources of 'transitivity' and 'reference' (i.e., Saussure's 'signification'), and both derive from the experiential metafunction, this is not a statement of two facts, but of one: the design of language has evolved in the living of life. The resonance of context and metafunction is a good indication of how eventually what 
happens as part of speaking (parole) might end up as a feature of the system of language (langue).

Fourth, the significance of the context of situation cannot be explained in terms of the material situation as such: the identity of the situation is created by its association with specific types of social practices. The material units of space and time are specialised by the ARC properties of types of social practice, giving them their identity; so 'classrooms' are rooms for teaching, the courts for dispensing justice, the shops for shopping, the banks for a variety of financial transactions, and so on. Nonetheless, the identifications of material spaces/moments are seldom completely 'binding'; other things can and do regularly happen in most locations: so the places mentioned witness the social practice of maintenance being cleaned and tidied up; and 'hold ups' sometimes occur in a 'bank', as do friendly chats.

Finally, social practice is as central to the development of culture as speaking is to that of language. Language is directly experienced only through its instantiations in texts; similarly, the working of culture becomes tangible to the social subject through involvement with the features of the social practices. Just as language is created, maintained and/or changed by social subjects participating in texts, so also are cultural institutions and domains, by the social subjects' participation in social practices. No wonder, the relations of language and culture and of text and situation as well as those of language and text and of culture and situation are so closely interwoven: deliberate and conscious enquiry is needed to understand these dense relations. Taking all this in consideration, the ARC hypothesis seems significant. But unlike the other three moves, this one, I believe, never came centre stage to be noticed by the SFL community in general: the publication (Hasan 2001) was not widely disseminated, and nothing incited me to actively try to foreground its potential. So, playing on the wording of a title by Goffman (1964) the ARC hypothesis, in fact, made no difference to the 'neglected context of situation'.

As I remarked earlier, one might wonder if context of situation is important enough to deserve such detailed attention. I believe it is, and I will return to this issue later (section Concluding remarks: the value of a scientific study of con/text). What the discussion shows is that underlying each move is one single issue, namely, that of precision - precision in the conceptualisation of the parameters, in the specification of their features, and precision in identifying their linguistic correlates. My serious move towards the paradigmatic description of context occurred in search of precision and accuracy: if the aim of the activity is the classification of register, and the success of that enterprise depends on achieving a precise means of identifying the correlates underlying that variation, then to me it was clear that the system based approach was required.

\section{The paradigmatic description of contextual parameters}

Paradigmatic description is not new to SFL; in fact it dates back to the birth of modern linguistics. The origin of the underlying concept can be traced as far back as Saussure's 'associative bonds' (Saussure 1966; 2006; Hasan, 2013; 2014). For SFL, in particular with its origins in Firthian linguistics, there had been a long tradition of orientation to this method (Firth, 1957; 1968; Halliday, 1961; Butt, 2001; Matthiessen, 2007; 2009; in press). Despite this rich inheritance, the system networks (henceforth 'sys-net') of today are rather different from Firth's, and they never had been anything like Saussure's presentation of 'associative bonds' (e.g., 1966: 126). Guided by a set of theoretical concepts, introduced mostly by Halliday in the 1960's to early 1970s, the paradigmatic method of analysis has 
moved a fair way - its representation in the form of sys-nets began in the early 1960's with Halliday. First applied successfully to the analysis of lexicogrammar (Halliday 196768; 1969), the method led to the metafunctional hypothesis in SFL (Halliday 1970a; 1973). Following the efforts by Halliday, the same descriptive orientation and mode of representation were further developed to describe the semantic unit 'message' in the early 1980s (Hasan, 1983, mimeo) which led to the development of other sys-nets, e.g., that of 'rhetorical unit' at the semantic stratum (Cloran 1994). These were employed successfully as research tools for the study of semantic variation (Cloran 1994; 1995; 1999; 2000; Williams, 1995; Hasan 2009b). To ignore the paradigmatic method in the description of context of situation would have been careless, particularly since the design of the system network is well suited to contextual parameters.

Each contextual parameter, representing a distinct area of human experience, is a variable that offers its own set of variant possibilities. And I hope to demonstrate below that the system network is an excellent resource for representing these possibilities in detail, thus maximising the chances of order and precision, qualities that the study of context is certainly in need of even today. Although the systemic description of contextual parameters per se is a nascent enterprise, a good deal of information is available in SFL on sys-nets in the domain of lexicogrammar and semantics and also in the domain of context (Butt 2004; Bowcher 2007; 2014; Cloran, 1994; 1995; 2000; Fontaine et al. 2013; Halliday, 1975; 1976; 2009; 2014, 2013; Hasan, 1983 mimeo; 1987; 1999; 2009b; 2013; Matthiessen, 1995; 2007; 2009; in press; Martin, 1992; 2013; Williams 1995).

\section{System networks as a language of description}

The sys-net conventions that help in producing systemic descriptions are dedicated to one specific goal, that of representing the analysis of semiological phenomena, but it is not easy to divide any units of the sys-net itself into two parts, i.e., 'the content', and 'its expression'. Options are perhaps the clearest example of content: presented in words, they can be interpreted as meta-linguistic terms naming elements of the description. But the system network has other resources for making descriptive meanings; its conventions - the lines, arrows, brackets, and braces whose arrangements constitute the material appearance of the sys-net - express meanings, since they stand for the relations of the descriptive categories.

\section{The technique of the system network: some basic concepts}

This section concerns some 'basic concept' for producing a sys-net. I would have much preferred to use throughout this section only the contextual sys-nets for illustrating the systemic notations. But the first figure, an exception, seems better suited as an opening.

'Point of origin' is a relevant concept, not simply because it is materially the first to appear but because it refers to the sys-net's object of enquiry: it is this 'descriptum' that will be described in every part of a sys-net ushered in by the point of origin. In Figure 1 this role is assigned to MESSAGE, and the ensuing sys-net are expected to describe the properties of MESSAGE (Hasan 2013), and nothing but MESSAGE. The descriptum, in SFL, is a theoretically motivated category: thus MESSAGE is a 'unit' on the 'rank scale' of semantics, with the implication that a good deal is already known about the identity of this descriptum. The work that the category does along with the others in the theory positions it unambiguously, its history having unfolded entirely by reference to the theory. Thus, 


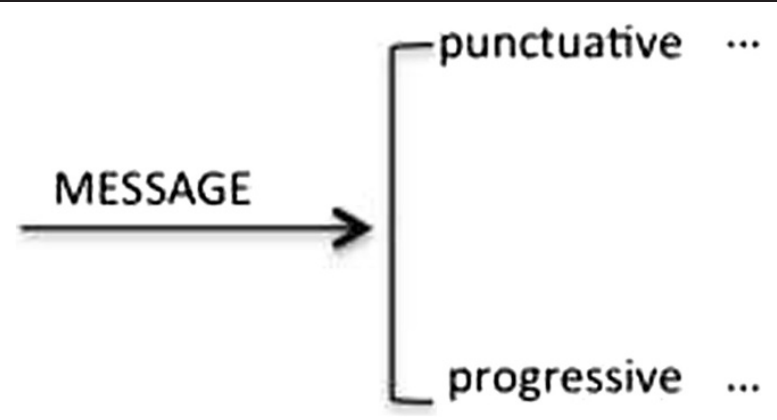

Figure 1 Point of origin and the primary system (source: Hasan 2013).

the identity of the semantic unit MESSAGE is fixed by what it relates to on the semantic stratum. The relation amongst the units on the rank scale of any languageinternal stratum is that of constituency: following from this, MESSAGE as a unit is made up of 'semes', and itself enters into the structure of a 'rhetorical unit' (Cloran, 1994). As a category at the semantic stratum it is itself construed by some categories of the lexicogrammatical stratum which lies immediately below it: the lexicogrammatical category, that typically realises the semantic unit message, is the unit 'clause'. At the same time, message as a semantic unit will itself realise certain features of the context of situation, which lies 'above' the semantic stratum. I will return to this discussion later to elaborate on 'realisation' in 'trinocular perspective'.

A quality of the point of origin is that as a term in a sys-net, its embrace, for want of a better term, is 'comprehensive'; it covers as much ground as may be necessary to describe the ultimate descriptum exhaustively. So, although Figure 1 presents only the initial system, the fact that the point of origin is MESSAGE implies that any sys-net describing any category of MESSAGE would be seen as relevant to it, either as (potentially) complementing it or as a critique or modification of the original. This is implicit in the fact that MESSAGE is a category in the theory. Point of origin thus announces a domain of relevant theoretical discussion: needless to say that the possibility of useful discourse will depend on the use of shared language. All the sys-nets pertaining to the same point of origin and considered valid in the theory are logically part of the same 'comprehensive sys-net'. This concept, like other theoretical categories, has 'no ontological status' (Firth, 1957: 181): most probably, it would be impossible to even display an image of a comprehensive sys-net exhaustively representing the description of its point of origin. It is conceivable that the digital media might provide an extensive and/or mobile surface for a display of the exhaustive representation, but even so the limits of human vision might restrict its perception as one single complete visual percept as one whole. SFL has always maintained that language is big, and it appears natural that linguistics, which describes language, should be bigger still.

The opening square bracket displaying the 'options' 'punctuative' or 'progressive' in Figure 1 signals a 'simple system'. The simple system can appear in two guises: it may have no company whatever, appearing alone, as does the system in Figure 1: these are known as 'individual systems'. By contrast, a simple system may appear together with at least one other simple system. This will be referred to as a 'simultaneous set', and each system of 
the simultaneous set will be called a 'member system' (=m-system). A simple system, whether acting as an $\mathrm{m}$-system or an individual one, must display more than one option. If only one option is shown in a system, the other being a zero or its equivalent, the system would be void: this is because an option is an option only if it is subject to true choice. The initial system of a sys-net, i.e., the first one to appear after the point of origin, is known as the 'primary system' of the sys-net, and will have the lowest degree (or 'level') of delicacy, which is to say that the information about the descriptum offered by the primary systemic options is at the lowest degree of detail. The last statements imply that the systemic options are able to 'offer' information of some kind. But where does this information come from and how did it get into the systemic option? The answer to this question unmasks the apparently inscrutable nature of the sys-net: I will describe this process briefly here, using as example only a succession of individual systems; later the description will be extended to more complex patterns of the sys-net.

The sys-net's process is 'declared open' with the display of its first system of options: in other words, nothing is given before that except the point of origin. The selection of the point of origin announces what the concern of that sys-net is, and the description will, in fact, 'reappear' as systems of related options, each system representing some property of the descriptum. For example, the information in the options of the primary system of Figure 1, 'punctuative' v. 'progressive', is nothing else than the possible properties of MESSAGE that the description has established at this early point. And the appearance of the first system with options is what constitutes the primary system. The two properties are mutually exclusive and together they are exhaustive of what can be said at this level of delicacy about the category MESSAGE: it will either be 'punctuative' (e.g., Hi!, you know what?, pardon! Wow!), or 'progressive' (e.g., didn't you talk to him? well, I wanted to, but I don't know where he hangs out and every time I go to his office he just isn't there...); and there will be no third category of MESSAGE at this level of delicacy. This may or may not be factually correct, but this is what the conventions of the sys-net are presenting as the analyst's analysis.

This account of the category MESSAGE specifies a relation basic to the sys-net's progress: this is known as the relation of 'dependency'. The disposition of a particular system depends on the category it is describing; the options of each system specify the make up of that category on which it depends. The steps following the primary move will essentially follow this principle with only one difference: since a simple system has more than one options, there will be more than one candidate for exploration. It is a sys-net requirement that for further exploration at any one specific point, one and only one option can be chosen from amongst the existing mutually exclusive options. This situation introduces the concept of 'choice' in the process of the sys-net's 'derivation'; and as just described the exploration of the chosen options 'reappears' as the options of the next simple system displaying, in its turn, a number of mutually exclusive options, ready to be chosen to continue further the cycle of the system's derivation. The origin of derivation was initiated with the point of origin; and the analysis of the chosen category as represented in the options of each new system is also taken to be exhaustive at that level of delicacy. It follows, therefore, that each next step in the process provides a more delicate analysis than the previous one(s): the information ever increasing in delicacy refers back to the ultimate descriptum, i.e., the point of origin. This, in a nutshell, is how a sys-net grows: it develops by further analysing the already analysed categories. 
Two related comments will complete this account: first, each simple system has two or more mutually exclusive options, each representing information specific to it but at the same time related to that of the other options in the same system. Typically all the options of one system bear some semantic affinity to the other(s), as evident from such examples as either 'private' or 'public', either 'masculine' or 'feminine', either 'graphic' or 'phonic', either 'negative' or 'positive', and so on. Secondly, for the description to be complete, every option of the said system must, in its turn, be chosen for analysis: no systemic option can be abandoned so long as there is anything to be said about it. As these 'coeval' i.e., belonging to same generation, options are analysed, the description is extended in the breadth of coverage, not in the depth/degree of delicacy. Of course, a stage can arrive in any network where an option might fail to follow the above 'cycle of birth and re-birth'; an option might simply terminate, while others are being further described. This can happen either because the 'terminal option' has reached the limit of its description: i.e., in the analyst's view no more need be said about it, or for some reason the analyst might suspend the analysis at that point. For example, the systemic options in Figure 1 are made only at the primary degree of delicacy, so it is not very likely that their description has been exhausted; their progress has been suspended here as of no further relevance. The measure of a sysnet's success is the extent to which it produces information in the shape of new options specifying greater descriptive delicacy at each new step, until the sum of these related choices has arrived at an adequate, explicit and explanatory account of the descriptum. Throughout, the conventions of the sys-net guard the transparency, orderliness and clarity of a process that has moved option by option, relation by relation, and system by system.

A few words on some terms as used here in talking of some sys-net relations. The term 'option' refers to the as-yet-unexplored property of a potential presented in the system: it is potentially 'choose-able'. 'Choice' is option selected for further exploration of a particular property; the analysis will develop by each choice used as an entry point into the next system ${ }^{1}$. And 'features' are properties of the unit under description, i.e., the sys-net's point of origin. These descriptive categories do not name 'entities': they actually refer to relations. For example, the point of origin has at least three kinds of relations: (i) as used so far, it is a unit at some stratum; (ii) it is an 'initial entry point' on which the first move in a sys-net depends; and (iii) it is the ultimate descriptum whose potential is to be presented in the sys-net as a continuous set of relations from the options of the system.

\section{The technique of the system network: the individual system}

In describing the mechanism of the sys-net development above, I focussed deliberately on the individual system in terms of the choice of simply one of its options at a time; being chosen enabled it to act as an entry point into the next individual system: this was referred to as the cycle of a system's birth and re-birth, leading to a new system. But the mutual relations of the entire set of choices from an individual system were not brought to attention. Figure 2 will repair this shortcoming, showing how the sys-net records derivations in full.

The main part of Figure 2 occurs on p. 42 (Butt 2004), but its history actually begins with the MODE of discourse ( $p$ 37) which specifies three terms in an opening brace, namely: (i) ROLE OF LANGUAGE; (ii) CHANNEL; and (iii) MEDIUM. These terms are labels for members of a simultaneous set of three systems. The m-system of MODE called ROLE OF LANGUAGE opens as an individual system (treated here as primary) with three mutually exclusive options: (i) 'constitutive'; or (ii) 'supported'; or (iii) 


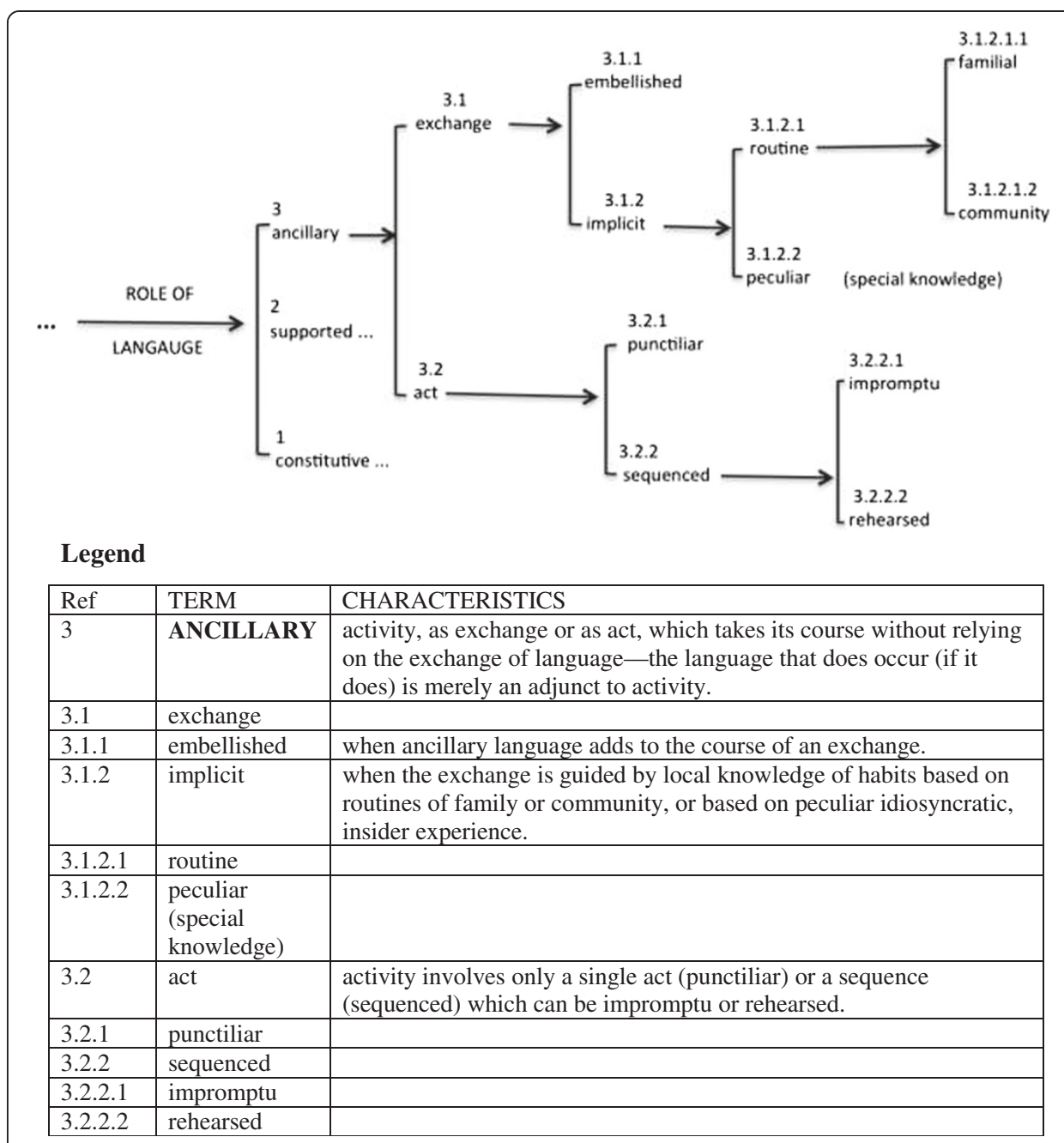

Figure 2 Mode: the 'ancillary' Role of Language (source: Butt, 2004: 42).

'ancillary' (p 38) ${ }^{\mathrm{m}}$. I have included, with apologies to Butt, this primary system of ROLE OF LANGUAGE in Figure 2 as presented here so as to indicate the dependency relations beginning with the primary option 'ancillary'; in other words Figure 2 as presented here is a combination of Butt (2004: 38 and 42). The fact that it is an individual system is signalled by the entry indicator, which is the arrow under the label ROLE OF LANGUAGE (Butt, 2004:38). The choice of the option 'ancillary' permits entry into the next individual system with two options of its own, namely, 'exchange' or 'act'. As Figure 2 stands, it signals that the options 'exchange' or 'act' are more delicate than 'ancillary'. The choice of the option 'exchange' in its turn acts as an entry point for a new individual simple system with two options 'embellished' or 'implicit'. Option 'embellished' is not chosen for further exploration; in the terminology used above, it is a terminal option. The choice of 'implicit' is the entry point for the next new individual system, and so on to the last systems. Figure 2 illustrates one method for charting paths in a sys-net which can be summed up as follows: so long as each system is individual and the relation is that of dependency, the cycle will take the form:

Option $=$ choice $=$ entry $\quad$ point $\rightarrow$ next $\quad$ systemised $\quad$ options $\rightarrow$ option $=$ choice $=$ entry point $\rightarrow$ option. 
Table 1 Legitimate selection expressions in Figure 2

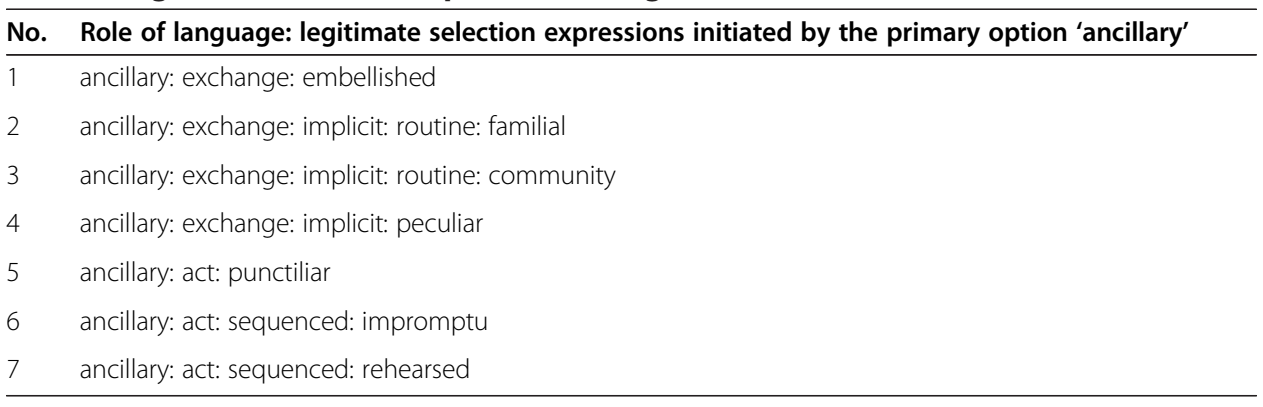

When a sys-net begins life at a primary option as 'ancillary' did in Figure 2, and moves system by system right through to the end, the traversal of the entire path formed by its relations is known as a 'selection expression' (SE); and each SE is a formulaic account of the properties of one sub-category of the descriptum, even though the name of the point of origin or its 'dimensions' may not be included in the SE itself ${ }^{\mathrm{n}}$. Obviously the lowest number of options in a simple system is two, and while there is no upper limit, typically simple systems are not found to go above four options. Further, the longer the paths, the greater the degree of delicacy: in principle, then, the maximum number of SEs can run to hundreds depending on how many option are found per system on average and how delicate the description is. So long as the relation is one of dependency throughout the sysnet, the 'legitimate' shape of the path will be as shown above. Though the SEs from the same sys-net may share some choices in common, as a whole, each SE will be unique. Table 1 presents the details of the legitimate SEs for Figure 2 depending on the primary option 'ancillary'. The colon between the two options signals the relation of dependency, i.e., in SE 1 'exchange' depends on 'ancillary' and 'embellished' on 'exchange'.

A system developed entirely by a cycle of dependence relation between individual systems, as here, resembles a simple taxonomy, with one notational difference that its branches move sideways. This mode of sys-net derivation is quite the commonest: in fact, most developed sys-nets will display a number of SEs built only or mainly by the relation of dependence. This type of system derivation is like simple taxonomies, which like the sys-nets can represent moves in delicacy. But sys-nets are also capable of representing more complex relations such as those of simultaneity.

\section{Complexity in systemic relations: simultaneous systems}

Systems occurring as members of a simultaneous set are probably not as frequent overall as systems occurring individually; but when they occur, they bring a multifaceted perspective to that chosen option which acts as their entry point, and they are also capable of forming the environment for complex entry conditions for new systems. I will discuss the latter relation below, using other examples of simultaneous sets, but on the following page I am going to begin with Figure 3, particularly because it raises certain issues, the exploration of which will allow some necessary elaborations.

As I see it, the issues here are: (i) the fourth primary system: what is it and why is it relevant in Figure 3? (ii) the first three primary labels listed as simultaneous truly speaking are not systems of FIELD, TENOR and MODE, since they have no options; what is their function here? (iii) The point of origin for Figure 3: context of situation $(=\mathrm{CS})$ is not a unit; so how is its identity established? On what ground can it be assumed that CS can be analysed in terms of the categories designed initially for the analysis of linguistic phenomena? 
I will address these issues below; but first a few words on how simultaneity of systems is signalled and other systems are derived from the options of the set of m-systems.

The simultaneous set in Figure 3 is signalled by an opening brace; here it holds four member systems; but any number greater than one may occur. The entry indicator for the simultaneous set is not an arrow but a plain line, linking the entry point and the brace of simultaneity. Simultaneity modifies the relation of the options across the m-systems: at any point only one option from each member system must be chosen simultaneously. All the options of the member systems in a simultaneous set must combine freely while maintaining the principle just described. If one single option depending on one m-system is chosen to act as the entry condition to the next system, the SEs should show not only that relation but the simultaneity relation to one option from each of the other member systems. This can be exemplified with the simultaneous set found in the system of

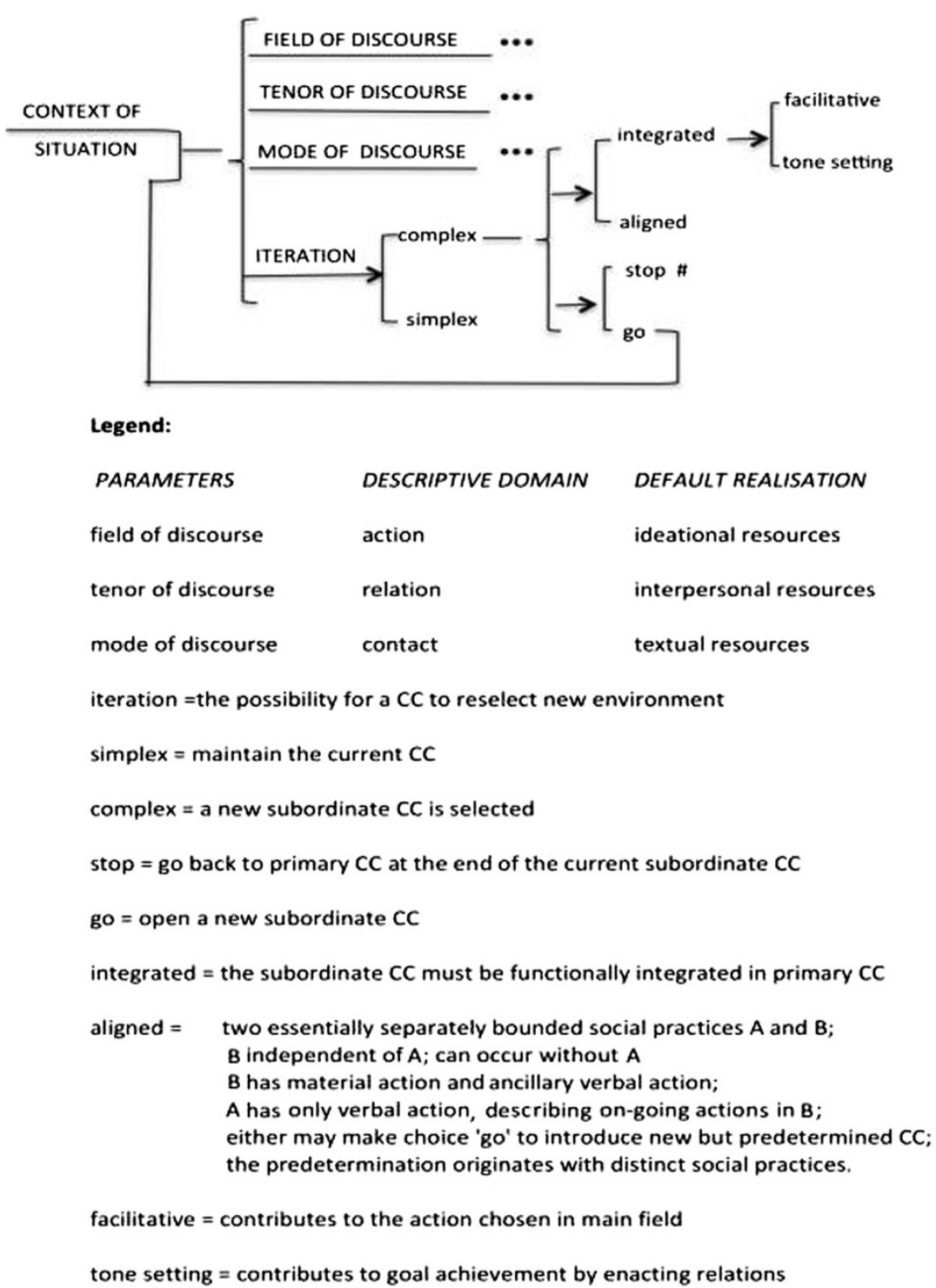

Figure 3 Context of situation as point of origin (adapted from Hasan: 2009: 182). 
Table 2 Choice paths combining $\mathbf{m}$-systems in ITERATION in Figure 3

\begin{tabular}{ll}
\hline 1 & simplex \\
3 & complex: integrated; stop\# \\
4 & complex: integrated; go \\
5 & complex: aligned; stop\# \\
\hline
\end{tabular}

Table 3 Selection expressions in ITERATION in Figure 3

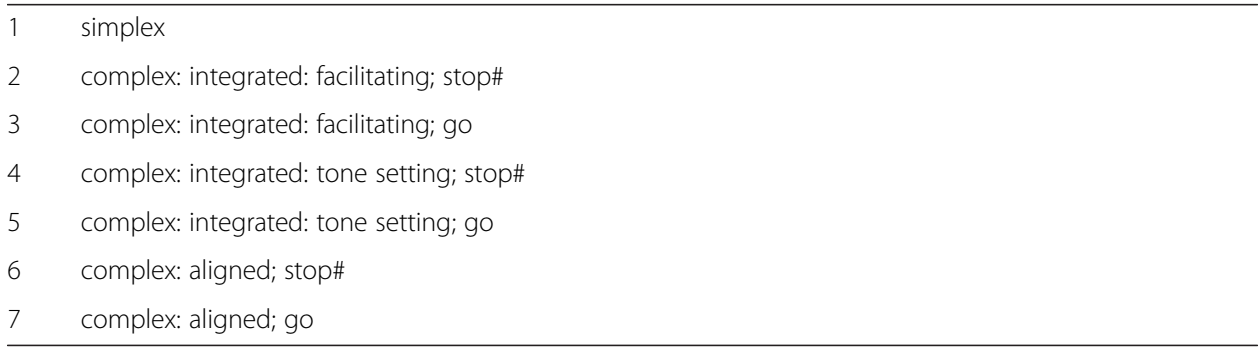

ITERATION in Figure 3, where the choice of 'complex' acts as the entry point to a simultaneous set consisting of two member systems, one with the options 'integrated' or 'aligned', the other with 'stop\#' or 'go'. Table 2 presents the selection expressions of the systemic paths as just described.

As before, the colon indicates a dependency relation, and the semi-colon, that of simultaneity of choices. If the option 'integrated' is chosen, allowing entry to options 'facilitating' or 'tone setting', the choices in the SEs will be as in Table 3.

As Figure 3 shows, 'integrated' or 'aligned' are options of the same individual system: so they can never combine legitimately. The options 'facilitative' or 'tone setting' depend on 'integrated'; so they can combine as shown in SEs 2-5 in Table 3. Had any options been shown for field, tenor or mode, they would be required to appear here as part of Figure 3.

I turn now to issue 1: what is the justification for including the system of ITERATION as a member of the simultaneous set in Figure 3? The less important reason is that it was convenient to use a small set of $\mathrm{m}$-systems to demonstrate the 'combination' of $\mathrm{m}$-systems. The more important reason is the function of this system in con/text description. Such an iteration system or something very much like it, was introduced as a systemic functional explanation of the difference between the concept of 'text complex' (Hasan, 1999: 246-273; 2000: 28-47) and Martin's 'genre combination'. The thesis is that a text complex is based on certain functional relations between the main text and the subordinate ones: the coselection of the latter accounts for the text's complexity. Unlike combinations based on contiguity, accidental or spatio-temporal, text complexity is not a happenstance: its explanation is in context, i.e., in the possibilities open to social practice. The primary system of ITERATION has two options here: 'complex' or 'simplex'. The choice of 'simplex' calls for 'no change' in the contextual configuration (CC) current at that point; the text no matter how long is a text simplex, as evident from the consistency of its registerial status. By contrast, the choice 'complex' implies some 'contextual shift'. I prefer to write 'con/textual' for the simple reason that there exists a 'solidary' relation between context and text. The choice of the option 'complex' acts as an entry point to a simultaneous set with two msystems. The first $m$-system from this brace specifies the function of the 
subordinate CC, which may be either 'integrated' into the main CC or be 'aligned' with it (other possibilities might be open, c.f. Hasan, 1999: 269). The options of the second m-system, 'stop\#' or 'go', specify whether iterative choice from the context of situation is to be made or not: if option 'stop\#' is chosen, the primary contextual configuration will be resumed putting an end to the sub-context; the option 'go' will imply that a con/textual shift is called for. The nature of this will be specified by the first m-system options. The choice leading to the selection of a sub-context would obviously mean re-entry into the systems pertaining to all three con/textual dimensions so as to access the necessary options relevant to the production of the new subordinate CC (for more detailed discussions, Hasan 1999). The signal indicating iteration of CS is the option 'go'. The line beginning at option 'go' makes its way to the point of origin, CONTEXT OF SITUATION. The left facing elongated or-bracket that reaches the point of origin is an iconic symbol permitting re-entry here via the point of origin into the simultaneous set.

I believe viable realisational statements relevant to the option 'integrated' have been presented in Hasan (1999: 246-273) and Cloran (1999). The option 'aligned' refers to paired social practice each pertaining to distinct domains which are systematically coupled as part of the realisation of the choice 'aligned'. This pairing is not an accidental co-occurrence, like washing up while discussing politics. Instead, it is culturally deliberate: one of the two social practices has the aim of describing what is going on in the other. Call the texts realising the two A and B. Given that the aim of A is to relay an account of the process of B (rather than 'replicate/summarise' it as might happen in 'note-taking' at a meeting), a syndrome of contextual features can be predicted in its CC. Thus in the FIELD of A the main action will be verbal 'constitutive'; any material action will be peripheral; the sphere of the FIELD will be 'specialised; institutional'; in TENOR, addressee will be 'absent: category: imaginary'; social distance between the speaker and addressee would be close to 'normal' degree; and in MODE material contact would be 'phonic, mediated, congruent: synchronic: single; public' - these choices together describe the register known as a 'sports radio commentary'.

B's contextual configuration will display considerably different choices: its FIELD will have 'focal' material action i.e., the action would be the centre of the social practice, largely involving physical expertise, and the sphere would be 'specialised; ... institutional' entertainment, and if there is any verbal action pertinent to the FIELD, it will be 'ancillary'; in TENOR social distance is likely to be 'close' and 'normal' with status as 'equal'; and in MODE material contact will be 'phonic; direct'. The social practice of sports, per se, is likely to be relatively independent and could occur in the absence of A: it does not have to be aligned; it is the choice 'aligned' that calls for the feature 'institutionalised'. However, if field sphere is 'institutionalised', as would be the case with (inter-)national sports, and other such public events, it is highly likely to be accompanied by the activity $\mathrm{A}$.

The contextual configuration underlying text type A, which is aligned to $\mathrm{B}$, is the register variety known as 'broadcast: commentary': during its production, any material action will be 'peripheral', the default choice being 'constitutive' verbal action (Hasan, 1999; in press a). With the choice of 'aligned', the two texts A and B are 'co-operating', being produced at the same time and standing in a logical relation. But unlike the 'integrated' texts, in this variety of text there is no primary text to which the subsidiary sub-text makes a 
contribution, i.e., integration and alignment lead to different kinds of textual complexity. The system of ITERATION appears to have a fairly wide range of application in SFL: it needs to be actually used to learn what other features might be relevant where and why. Finally, iteration is not specific only to complexity in con/texts; all language internal units at the content plane manifest the possibility of appearing as simplex or complex. However, the same symbol as presented in Figure 3 is used in all cases to represent iteration: the details referring to the other options will vary from those shown in Figure 3, but the actual iteration system with the options 'stop\#' or 'go' together with the symbol for iteration will be the same at all the other strata. In further discussions of Figure 3, the system of ITERATION will be largely ignored.

The second issue arising from Figure 3 is the absence of systems for three of the entries: the entries simply indicate that apart from the iteration system, three other $\mathrm{m}$-systems will form part of the displayed simultaneous set. The lines below the label indicate that the anticipated systems will themselves be m-systems; but no systems of options are presented. Let us imagine now that Figure 4 joins Figure 3 just where FIELD OF DISCOURSE is forecast.

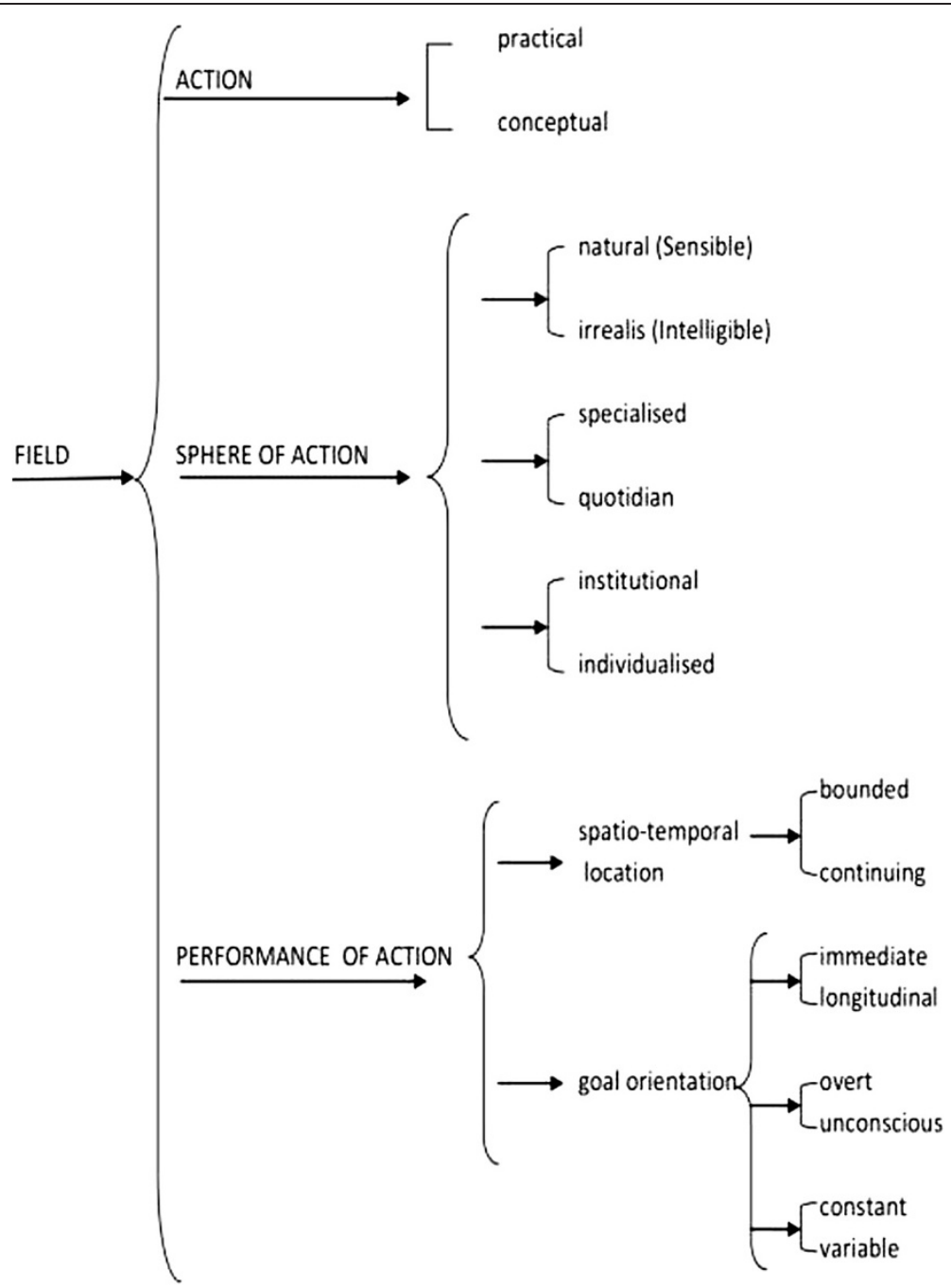

Figure 4 A Field network (Source: Bowcher, 2014, 21(2): 176-209). 
Positioned thus in Figure 3, Figure 4 will display the initial steps missing from it: it now has a point of origin and it is clearly an m-system in a simultaneous set where two others systems - tenor and mode - are also anticipated. At the same time Figure 4 brings the gift of primary systemic options to Figure 3, confirming that the primary system of FIELD is indeed complex. According to Figure 4, the primary system of FIELD consists of (i) ACTION, (ii) SPHERE OF ACTION, and (iii) PERFORMANCE OF ACTION ${ }^{\mathrm{P}}$. The complexity of FIELD is indicated by the form taken by these three primary systems, each of which is in the last analysis a member of one simultaneous set. The simplest to interpret is the system of ACTION with just one individual system with two options. The second system, called SPHERE OF ACTION, consists of a simultaneous set with three m-systems. The third primary system, called PERFORMANCE OF ACTION, is also shown as a simultaneous set with one of its member systems, called SPATIO-TEMPORAL LOCATION, appearing as an individual system while the other three make up a simultaneous set called, GOAL ORIENTATION. We thus have two layers of simultaneity. Figure 5 schematically represents the union of Figure 4 with Figure 3.

This description of field is formally equivalent to a set of eight simultaneous systems, each consisting of two options. So, notwithstanding the repeated nesting of simultaneous sets, all these systems would be treated by the conventions of the sys-net as mutually combinable. It is possible to preclude free combination, for example by using the 'if-then' relation indicated by what is referred to as the 'arrow diacritic' below (section Crossreferencing in sys-nets: relation based diacritics). In the absence of that preclusion, the

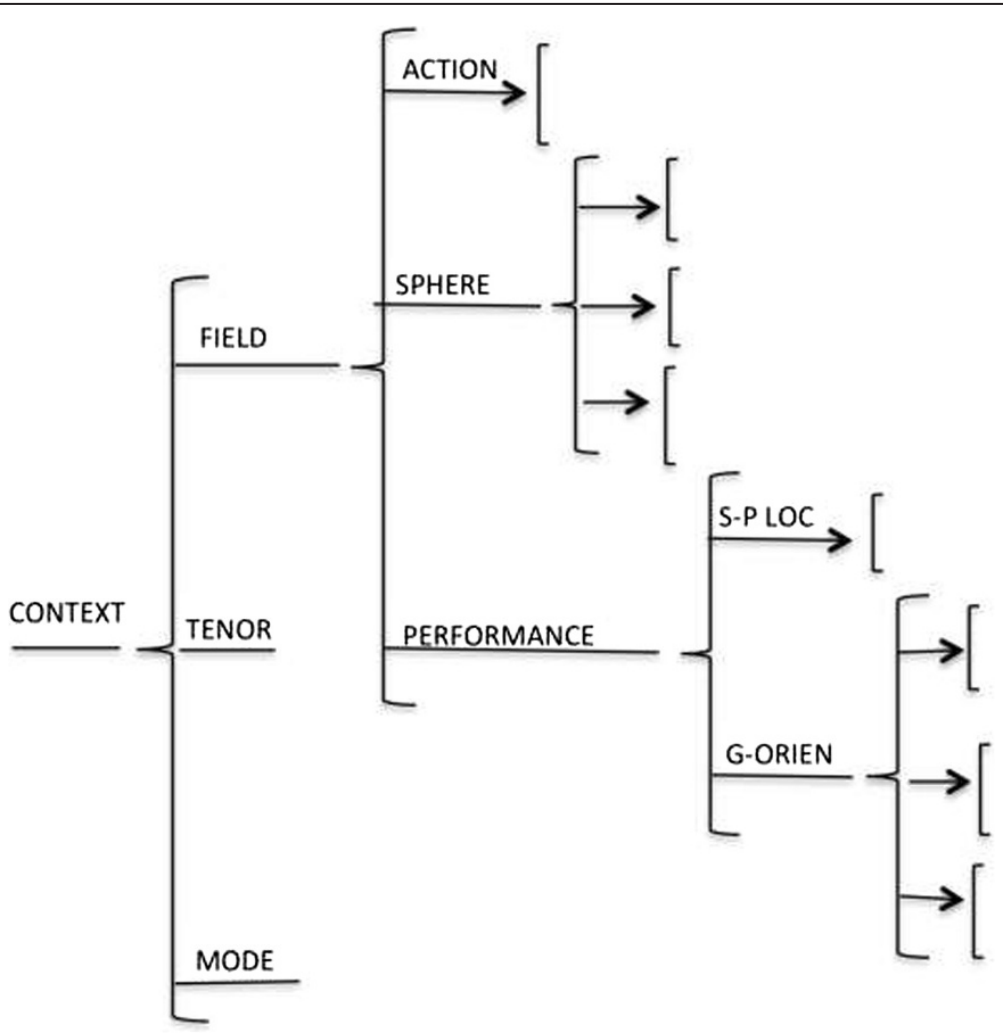

Figure 5 A schematic network: the union of Figures 3 and 4. 
number of selection expressions produced by the concurrent systems of field will be $2^{8}$, or 256, each describing one particular primary sub-category of FIELD.

Turning now to the third issue: there is no doubt that context of situation is a descriptum in search of a description; but it is also true that context is not a unit of any kind in language $\mathrm{q}^{\mathrm{q}}$. For linguists to explore this domain might smack of doing 'spontaneous sociology' (a practice Bourdieu, 1991: 37-38 criticised while himself presenting a case of 'spontaneous linguistics'). It is worth pointing out, though, that the linguists' context is neither the physicists' 'real' world, nor the geographer's, nor yet the sociologists'; it is quite a different abstraction from that same 'reality'. And there exists a valid basis for linguists to attempt this abstraction, since it constitutes the context relevant to human verbal interactions. Members of a speech community act and interact with each other in some environment concerning some aspect(s) of that environment. Although there is a good deal more to the vast universe of social and material 'reality', linguists need to understand aspects of that human environment, summed up as 'who says what to whom where when and why'. The linguistic interactions we engage in do, however, bring changes to aspects of non-linguistic reality, which suggests that the boundaries between language and social reality are, in effect, not water-tight: there must exist some way for social reality to penetrate into language, and vice versa - our locutions change the material, whether temporarily or otherwise, and the concept of metafunction shows how the outside enters the inside of language.

In SFL the reciprocal nature of this relationship has been recognised at least since Firth. Halliday (1991/2007b) used the concept of realisation in modelling the symbiotic relation: he suggested that language and culture are analogous human resources: and the system of language is related realisationally to the context of culture just as text as language use is related to context of situation. Moreover just as the system of language is instantiated by the various text types of a community so also culture is instantiated as situation types. An adequate model of language needs to explain the nature of language both as system/langue, i.e., as a web of associative bonds, and as language use/parole, i.e., as a form of interrelated linguistic acts. Such a model must probe the systemic nature both of language as a semiological system and of cultural practices as the impetus that has lead to relevant, context based, text production. The perspective adopted in SFL on the text's context has demonstrated that far from being un-generalizable, parole, so viewed, presents a functional and orderly heterogeneity; and the SFL view of their relations has led to better understanding of the centrality of cultural environment in the formation of individuals. The interactants as the producers of the community's texts are persons who, throughout life, continue to internalize ways of saying and meaning that are specific to occasions of talk recognized in their culture. The strength of SFL's reasoning is based on its analysis of linguistic and cultural processes: so, for example, the hypothesis of context metafunction resonance (CMR) shows a more robust understanding of the mutual relations of language and reality than the established notions of truth, reference and correspondence. The concept of realisation as developed in SFL has demonstrated the reach and efficacy of language events, thus justifying the recognition of context as a language external stratum of the linguistic theory.

To establish the identity of the context, one needs to focus on the varying features in the textual environment; this is what register variation correlates with: this was the basis 
for the recognition of the three parameters whose linguistic character was made more specific in the CMR hypothesis: now field, tenor and mode could be viewed as realisationally oriented respectively to the ideational, interpersonal and textual meaning-wording. And this can be interpreted as assigning each parameter a 'default realisation' thus granting the possibility of 'variant' realistions under specifiable conditions. The ARC hypothesis strengthens the basis of the parameters by a systematic correspondence: for example, field concerns what concerns Action, so it should subsume aspects of Action, e.g., its types, circumstances and participants as recognized within the culture; and of course these are exactly the phenomena, realised 'more often than not' as meaning-wording derived from the experiential metafunction. The same applies mutatis mutandis to the other two parameters.

Yes, context of situation as point of origin differs from 'message' or 'clause': but, perhaps with ARC in view, the unity of context is not subject to doubt, nor is the identity of three parameters that underlie the metafunctional hypothesis. The parameters are not like the units on a rank scale, but, significantly, they share some characteristics of metafunctions. In SFL, there is no metafunctional hierarchy, whereby the ideational is 'raised' to the status of semantics as in many linguistic models, while the other metafunctions constitute the subject matter of 'pragmatics': the latter had begun its life as the study of some language patterns but somehow those language patterns seem to have fallen outside 'linguistics proper'. By contrast, in SFL, the three metafunctions are alike and contribute jointly to the description of linguistic units. This, however, does not mean that some particular segment of the units can be isolated as realising one particular metafunction. Similarly the text's meaning-wording realises all three aspects of the context, but no specific part of the text can be singled out as realising just field and nothing but field; and the same applies to tenor and mode as well.

Notwithstanding good reasons for treating context as point of origin, its in-built complexity cannot be ignored. Would this affect its linguistic description? At first glance, the use of multiple braces may appear iconic of that problem. But somewhat surprisingly even here it seems possible not only to generalize about the function of the multiple braces but also to offer analogous relations at the language internal strata of wording and meaning. Thus, the first large opening brace of a sys-net such as incorporated in Figure 3 seems to have an especial function: occurring immediately after the point of origin or close to the primary system - but always in the vicinity of the less delicate part of the sysnet - the brace seems to be reserved for identifying the metafunctional orientations of the various systems that will constitute the comprehensive system network pertaining to the point of origin. For example, with the unit 'clause' as the point of origin, the primary system has two options 'minor' or 'major'; and following 'major' an opening brace announces a number of dimensions such as those of mood and modality (interpersonal orientation), transitivity, tense and projection (ideational orientation), and theme and information focus (textual orientation) (see Halliday, 1976; Matthiessen and Halliday 2014 etc.). Similar to this, at the semantic stratum, Figure 1 has shown two primary options, 'punctuative' or 'progressive'; the latter option is followed by the first large brace of that sys-net; and this specifies four entries, each oriented to a specific metafunction: the first to interpersonal, he next to textual; the third to logical and the last to the experiential metafunction (Hasan 2013: 286). A similar situation unfolds with context as well: thus, in Figure 3, immediately after the point of origin, is the first brace; it announces the four 
domains pertinent to the description of context - that is to say, the three dimensions of field, tenor and mode plus that of iteration. I interpret this as signifying that these three domains have carved up the vast area of description called context of situation by reference to their metafunctional orientations.

The actual systems open when each of these sub-domains announces its relevant 'entry points': these are displayed at this point as ACTION, SPHERE OF ACTION, and PERFORMANCE OF ACTION; and each of these is followed by the primary system(s) with options as in Figure 4. This pattern is found in much of the work presented to date (e.g., Hasan, 1999: 311; 2009a: 183, Figure 3c; in press a; Butt 2004; Bowcher 2014, c.f. Figure 4 here).

\section{Complexity of systemic relations: complex entry conditions}

In the above discussion of sys-net derivation all the entry points have been simple: in each case the choice of just one option at any one time has acted as the entry point to the next system. A simple entry point is perhaps the most frequent, but sys-nets at each stratum of the content plane also display what is known as 'complex entry point': here not just the choice of one but of two or more options from different systems is needed. There are two types of complex entry points: the 'conjunct' and the 'disjunct'. I begin below with the complex conjunct entry point; but a disjunct entry point will be encountered in the course of discussing the systems introduced by the conjunct entry condition. Like the simple entry point, the conjunct entry may either allow entry to one individual system or to a simultaneous set with two or more m-systems. Figure 6 presents an example of all the features just discussed.

Figure 6 is a fragment of the paradigmatic description of the MODE OF DISCOURSE (MD) which is shown as the third contextual parameter in Figure 3. MODE, as Figure 6 shows, is altogether and only concerned with contact, the two dimensions of which are MATERIAL and SEMANTIC. This concept of MODE reflects a universal characteristic

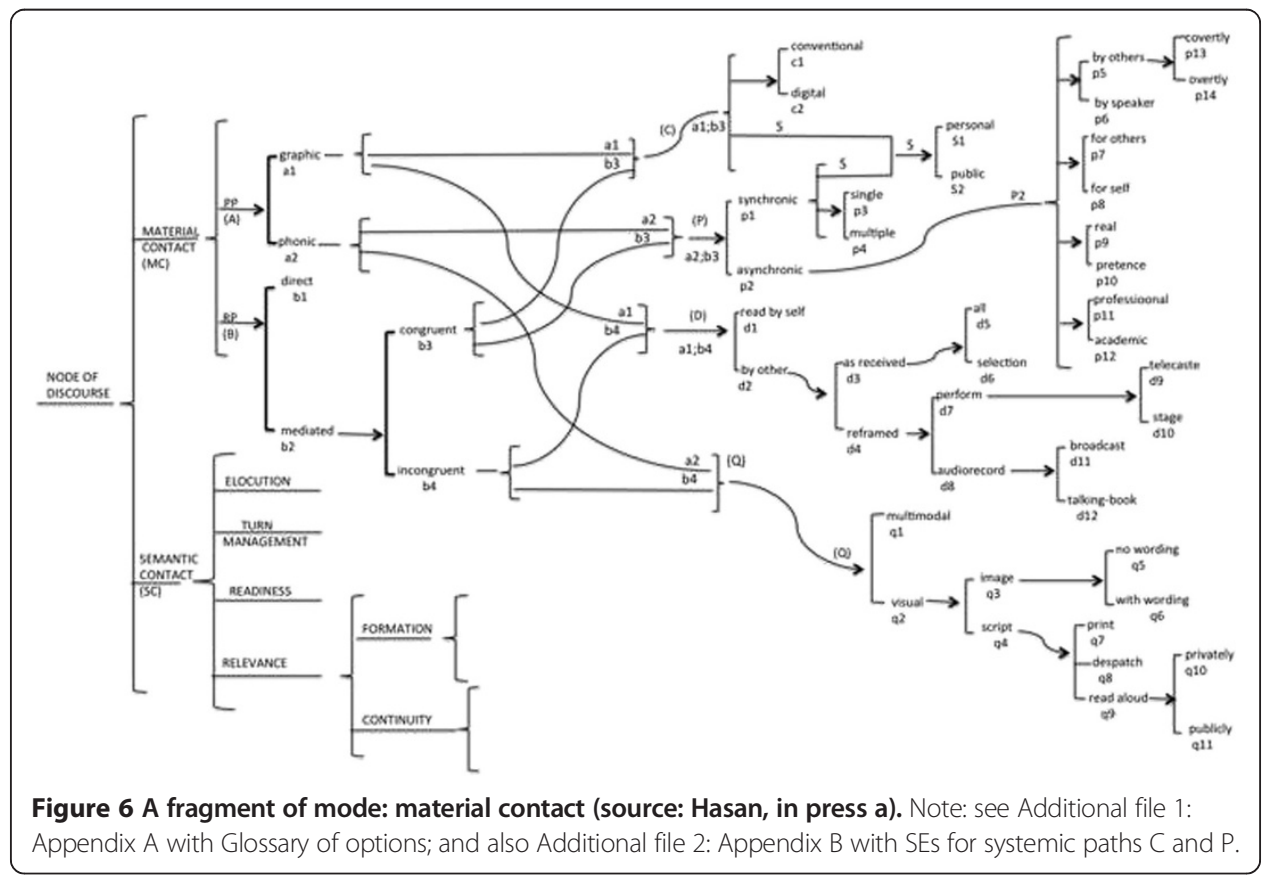


of the semiological unit (the 'sign'): it stands for something other than itself - this is its 'signified'/ meaning - and its shape, i.e., the 'signifier', must be formed of some substance which allows the human body to sense it. The sign system of language is one such semiological system, and speakers typically encounter it in the course of 'doing things with words'. Activities of this kind, by definition, depend on the exchange of meaningwording. This can only occur if meaning-wording can be 'transduced' into some sens-ible matter thereby allowing the body-brain-mind to access that which is being signalled, i.e., the meanings (c.f., discussion of 'realisation' in section Realisation: the renewal of connection with experience below). In Figure 6, MATERIAL CONTACT (MC) is an attempt to describe the possible means of expression that normal human beings can use in producing and receiving meaning-wording. The systems of SEMANTIC CONTACT (SC) concern the description of intellig-ible aspects of language, which would primarily facilitate access to those meanings whose choice will facilitate continuity, coherence and textual organisation. [see Additional file 1: Appendix A re Figure 6 options].

As a primary semiological system (Hasan, 2014), by definition, language was an 'embodied' resource, that is to say, meanings were circulated entirely by bodily resources. However, today, 'graphic' relay, i.e., the technology of producing symbols on a surface, plays an undeniably prominent part in human life. This has most probably happened by language appropriating writing to such an extent that the words 'written/ spoken' no longer refer to just two modalities of expression: they actually name two styles of meaning-wording. The written style is typically found in communication that neutralizes material distance while the spoken foregrounds face to face interaction, or direct access to sound waves. Although speakers are sensitive to variation in the properties of expression, as is obvious from their evaluative perceptions, such as accent variation (Abercrombie, 1965) or the 'sing-song' way of speaking, the linguistic styles, known as spoken/written, cannot be analysed by focussing on modes of expression alone: these, by way of their realisation, mostly depend on the features of meaningwording, which means the styles in question would be the realisation of options described by the systems of SEMANTIC CONTACT. These interconnections between systems of semantic and material contact are not described in Figure 6, which is what makes it a tentative fragment of MODE: it is highly likely that when those aspects are explored, the systems both in $\mathrm{MC}$ and SC will look somewhat different from Figure 6. Meanwhile it seems that using 'graphic'/'phonic' to refer to MC resources will distinguish between expression and content as two distinct modes of establishing contact.

MC opens with a simultaneous set consisting of two primary m-systems, PRODUCTION POINT (PP) and RECEPTION POINT (RP), with two options in each: the PP options 'graphic' (a1) or 'phonic' (a2) refer to relay, and the RP ones 'direct' (b1) or 'mediated' (b2) to means of Receiver access. In 'direct' access no third agency - man or machine - intervenes: so the accessed 'text' reaches the addressee in its original form. The bodily means of access are obviously visual if relay is 'graphic' and aural if it is 'phonic'. When 'phonic' relay is used at production point, the 'direct' access at reception point will be more or less synchronic; however, unpredictable degrees of time lag will occur in the case of 'graphic; direct' (more on this below). The option 'direct' is shown as 'terminal'; but the choice of the option 'mediated' allows access to a simple system with two options 'congruent' (b3) or 'incongruent' (b4). Since the PP and RP systems form a simultaneous set the legitimate choice paths for these would be as shown in Table 4 . 
Table 4 Legitimate choice paths of primary options in MATERIAL CONTACT in Figure 6

\begin{tabular}{lll}
\hline No. & Legitimate primary choice paths in $\mathbf{M C}$ & \\
\hline $\mathbf{1}$ & $\mathrm{a} 1 ; \mathrm{b} 1$ & graphic; direct \\
$\mathbf{2}$ & $\mathrm{a} 2 ; \mathrm{b} 1$ & phonic; direct \\
$\mathbf{3}$ & $\mathrm{a} 1 ; \mathrm{b} 2: \mathrm{b} 3$ & graphic; mediated: congruent \\
$\mathbf{4}$ & $\mathrm{a} 2 ; \mathrm{b} 2: \mathrm{b} 3$ & phonic; mediated: congruent \\
$\mathbf{5}$ & $\mathrm{a} 1 ; \mathrm{b} 2: \mathrm{b} 4$ & graphic; mediated: incongruent \\
$\mathbf{6}$ & $\mathrm{a} 2 ; \mathrm{b} 2: \mathrm{b} 4$ & phonic; mediated: incongruent \\
\hline
\end{tabular}

The option 'direct' cannot combine with 'mediated': being options of the same simple individual system neither these choices nor their derivative options can ever combine the option 'direct' or any options depending on it. Each of the six choice paths above could in theory be chosen to function as a conjunct entry condition; in actual fact, only choice paths 3-6 are shown as functioning in this capacity. [see Additional file 2: Appendix B re C and P Choice paths in Figure 6].

The signal for indicating a conjunct entry point is complex. It involves small braces and connecting lines to signal the participating options, as well as the entry point where the move to the next systems occurs. Figure 6 clearly displays the formation of this signal. A new address has been given with a capital letter to each indicator of a conjunct entry point; the same address is continued but in the lower case for the system introduced by the fours conjunct entry points. The first such entry shown in Figure 6 involves options 'a1; b3' and its own address is (C) so the options in one of its $\mathrm{m}$-system are $\mathrm{c} 1$ or $\mathrm{c} 2$; a similar arrangement indicates the remaining three conjunct entry points. These details are carried by the conjunct entry indicator which is shown as a line IF followed by a simultaneous set (as in C), or an arrow IF the next system is an individual (as with $\mathrm{P}$ ). This shows the complex conjunct entry points may allow access to either an individual system or to a simultaneous set.

Choosing options to form a complex conjunct entry point is subject to a principle: for example, none of the options related to the same primary system can combine; it follows that in Figure 2, no conjunct entry point can be formed. The most hospitable environment for a conjunct entry point is from the m-systems of a simultaneous set. This of course does not mean that the options are bound to do so: an options of the m-system can be chosen to act as a simple entry, as if it belonged to an individual systems. However the principle stated earlier will apply: that one and only one option from each m-system must be chosen at any particular point. If each derivation is simple, there is expected to be a huge explosion of SEs. It is, however, possible to relax the last principle IF the choice made is such as to prevent free combination or 'default' choices are shown to apply. In any case, the two ways of deriving sys-nets from the m-systems of a simultaneous set - either by simple dependence or by the complex conjunct entry points - provide different information about the ultimate descriptum. This makes sense, since, as described above (The technique of the system network: some basic concepts), each simple system views the chosen option from one distinct perspective, whereas the complex entry points do so by reference to multiple options either in conjunction or in disjunction, and each combination differs from all the other possible ones as the choice paths in Tables 3 and 4 show. Below I pursue the formation of two conjunct entry conditions $\mathrm{C}$ and $\mathrm{P}$ to illustrate these points, and 
in the course of doing this I will also introduce the operation of a disjunct entry condition.

The entry $\mathrm{C}$ whose options are 'graphic; mediated; congruent', gives access to a simultaneous set, the first member of which has two options 'conventional' (c1) or 'digital' (c2), the second system has 'personal' (s1) or 'public' (s2). Since, 'graphic' relay is used, typically when the addressee is 'absent' (see Figure 7: esp. textual roles), some time lag between relay and reception is inevitable. With 'direct' relay, ideally no mechanical or human agency intervenes between relay and access, but this is what must happen in 'mediated' reception. The options 'digital' or 'conventional' indicate the nature of the intervening agency: the option 'conventional' refers to the use of such older methods as handwriting, typewriting, or print (at PP), mediated access to which is possible by mechanical mean such as post, print etc. The second option 'digital' (c2) refers to more recent modalities (though its reach is growing fast). But can all these modalities be used anywhere by anyone as the means of graphic relay? This question is answered by system $S$.

If the addressee is known to the speaker as a 'person', the option 'personal' (s1) will be the default choice, while the second option 'public' (s2) will be chosen if the addressee represents a communally defined 'category' (see Figure 7 for these options). Because the systems $\mathrm{C}$ and $\mathrm{S}$ are simultaneous, the 'conventional' or 'digital' modalities of access will combine with 'personal' or 'public'. In contacting a 'personal' addressee, such as a friend, relative, close colleague, or neighbour, the choice of handwriting is most likely to be used as a 'conventional' means of relay, as in writing letters, memoranda and quick informative notes. By contrast 'public' implies intervention by some agency or organisation facilitating physical access, e.g., the postal service, use of hand delivery by a third party, use of a notice board, and so on. Notably, with the options 'conventional (c1); personal (p1)' the speaker retains control on the range of his text's dissemination. This control is lost with the choice of the option 'public': the speaker's text is addressed to a communally recognised 'category' members of which are not necessarily known to the speaker personally; and with this will vary the form of the graphic modality. Instead of handwriting, other 'conventional' but more formal means will be typically employed for material contact, such as print; somewhere in between (published) print and handwriting are the 'in-house' printing facilities such as typewriting (now nearly obsolete) or a home printer. When the text has the choices 'graphic; mediated: congruent: conventional; public' the relayed message can be accessed by anyone: the speaker has little or no control over the text's range of dissemination; and anyone with physical access to the material form of the message can 'become' a receiver, irrespective of the addressee type which had originally guided the speaker's production of meaning-wording. The constraint 'personal' or 'public' is applicable to the 'digital' means of mediation as well: if the option 'personal' is coselected with 'digital', i.e., material contact is with friends, colleagues, neighbours, and so on, then typically the 'digital' means of emailing is the most probable, though with the rapid adoption of technology the situation is considerably fluid. The 'public' face of this digital modality of presentation is perhaps the Facebook, Twitter, Blog, and such like. As Halliday has often reminded us, the description of language is always open-ended, and more can be said on this topic e.g. about the aesthetic function of calligraphy, or the visual lay out of graphic material and so on. But the more 
pressing question for this contribution is: where did the system S come from? In answering this question, I enter into a brief account of the complex disjunct entry point.

I have shown that the options of $\mathrm{m}$-system $\mathrm{S}$ combine systematically with those in the $\mathrm{m}$-system $\mathrm{C}$, which implies that system $\mathrm{S}$ has been derived in response to a more delicate analysis of the choice path 'graphic; mediated: congruent'. This is true, but it is not the complete story of the system S, which straddles two distinct simultaneous sets, one to which access is allowed by the conjunct entry point $\mathrm{C}$ as discussed above, and the other to which entry is allowed by a simple entry point, namely the choice of the option 'synchronic' (p1) in the system P, which was itself introduced by a conjunct entry point. These two choice paths, $\mathrm{C}$ or $\mathrm{P}$, cannot be combined at any point in this sys-net: their choice paths began with the mutually exclusive options 'graphic' (a1 in PP) or 'phonic' (a2 in PP) belonging to the same primary system of PP (compare the analogous choice paths 3 and 4 in Tables 3 and 4 above). But despite this incompatibility, the options of the $\mathrm{m}$-system $\mathrm{S}$ are relevant to both. This is the sort of environment where a disjunct entry point is most likely to occur: this type of entry condition calls for at least three simple systems working together. In the case of $\mathrm{S}$ that allows disjunct entry, choices from two other systems $\mathrm{C}$ or $\mathrm{P}$ are involved: at any one point either one option from system $\mathrm{C}$ or one from system $\mathrm{P}$ may combine with one option from system $\mathrm{S}$, and the elongated closing bracket with one foot in $\mathrm{C}$ and the other in $\mathrm{P}$ signals this 'either/or' requirement. Though perhaps less common than the conjunct entry condition, instances of disjunct entry point are found both in lexicogrammar (c.f., 2014, 2013, the system of MOOD) and in semantics (c.f., Hasan, 2009b; 2013, the system of ROLE EXCHANGE).

System P, like system C, is introduced by a conjunct entry point consisting of the choice 'phonic; mediated: congruent'. With the choice of 'phonic' at production point, there will be hardly any time lag between the 'oral' relay and 'direct' 'aural' access. But the possibility of disruption in this normal pattern becomes a significant issue at reception point when the choice path contains the options 'mediated: congruent', as it does for P; this is because mediation always leads to the text's 'displacement' from its point of production, and this normally involves the intervention of a mechanical and/or human agency other than the speaker. The options of the first system derived from the conjunct entry P focus on this issue: the option 'synchronic' (p1) implies no noticeable time lag, while 'asynchronic' ( $\mathrm{p} 2$ ) implies that there will be delay: this latter option materialises as an act of recording, and it is not pursued further in this article.

Given the nature of the phonic relay, some mediating technology will obviously have to be used in order to receive the message aurally at a distance in time and space. The option 'synchronic' acts as an entry condition for a simultaneous set with two $\mathrm{m}$-systems. One of these is the continuation of $\mathrm{P}$ and its options 'single' or 'multiple' refer to the means whereby the 'synchronic' and 'congruent' access of the phonically relayed message is maintained: the option 'single' (p3) implies 'phonic' relay at PP which is heard as if within ear-shot; the text is accessed aurally without any time lag at the moment of being produced. This enabling device is the telephone, by which the message becomes near-synchronic, yet mediated; and only a single modality is at work in accessing the message. By contrast, the option 'multiple' would involve some technology such as Skype: here in transmitting the relayed phonic modality visual access is also enabled, and that allows the interactants to access some other aspects of each other's material situational setting, 
including the kinesic. The second $\mathrm{m}$-system is the familiar system $\mathrm{S}$, with one 'foot' in this simultaneous set and the other in that derived from entry point $\mathrm{C}$. The options of system $\mathrm{S}$ are already familiar: as before, in general terms, their concern is to specify the constraint on the use of the available technological devices, but with some subtle differences. So when the option 'personal' is co-selected with 'single', this refers to the use of the telephonic device with the single modality of speaker's voice: here, 'personal' refers to both who relayed the message and also who received it; the interactants producing the text are the ones who have access to the text. The combination of 'public' and 'single' refers to a 'live broadcast', as for example with sports commentary, where the receivers may not be known to the speaker; the speaker is not personally addressing a targeted addressee. When 'multiple' and 'personal' combine, then reception is mediated by a technology that allows more than one modality for accessing the message, simulating a face to face direct relay. By contrast, when the features 'multiple' and 'public' are co-selected, the difference is not so much in the modality of access as in the meaning-wording of the message (i.e., in semantic contact); with the choice public the social distance between the speaker and the distant receiver is likely to be 'somewhat' 'distant' (c.f., Figure 7).

Much of what has been described by the options of the two simultaneous systems in Figure 6 goes beyond just the modalities of relay or access; the difference between the 'personal' and the 'public' mode of access is also a difference between talking to someone 'fairly' 'close' or 'distant'; the realisations is likely to extend to the text's meaningwording. For example, we know that with the option 'a-synchronic', when retaining aural access at a distant point from relay, the message must be audio-recorded, and that data orally presented and audio-recorded is affected seriously by 'noise' of any kind. This issue has not been raised in this paper. The complexities of 'phonic' expression reappearing as 'phonic' after being 'mediated' clearly go beyond what is indicated above (see the options p5-p14). If some 'real' phonic data is recorded 'by others' 'for others' in carrying out a 'professional' duty, this may often be straight forward, e.g., a person in charge records the proceedings, as often happens on official occasions such as symposia. But suppose the despatch of the message to an addressee is professionally required, and someone records that message on the dictaphone: here the phonically relayed message would be received by that intended addressee in 'graphic' not in 'phonic' mode. This possibility could be described by some options in Q (dealing with 'incongruent' access). What is missing is an intermediate stage: so far as this message is concerned, at production point it is 'phonic' but it is also 'mediated: congruent: a-synchronic'. So the message is recorded and as a recording it would be accessed only by a clerical staff member, who is clearly not the addressee but the person in the middle who transcribes. The possibilities of what can happen to a recorded message are about as varied as are those of 'graphic; mediated: congruent: public', whether 'conventional or digital'. So the description as presented in P, though probably not incorrect, could be improved. There appears to be a need for every parameter to recognise the relevance of the others. But is there any sys-net convention to enable this cross referencing?

\section{Cross-referencing in sys-nets: relation based diacritics}

Cross-referencing has been in practice from the early days in the use of the sys-net (e.g., Halliday 1973: 47; Kress 1976), typically by use of what I am referring to as 'diacritics' 
since they consist of signals attached to option(s) or, in some cases, to specific entry point. Their function is generally to signal relations that are inter-stratal, though the relations themselves are either 'inter-systemic' or 'intra-systemic'. Both may be viewed as a kind of shorthand, since either type can actually be replaced by some more 'wiring' (i.e., connecting with lines and braces). Diacritics are preferred as making less demand on space and energy. Figure 7, representing the description of tenor of discourse, shows both kinds of diacritics. Here only those options are discussed that are relevant to the relation of the diacritics. [See Additional file 3: Appendix 3 for the glossary of options in figure 7].

Figure 7 concerns tenor of discourse. This is conceptualised as interactant relations; and the features pertaining to every system here are either complementary or reciprocal. The sys-net, in principle, consists of three simultaneous dimensions along which INTERACTANT RELATIONS are organized: the AGENTIVE RELATIONS, built around the social practice they are engaging in here an now; the TEXTUAL RELATIONS, the considerations around which their meaning-wording roles are calibrated; and the SOCIAL RELATIONS, consisting of their interactive biography and their social positioning. Figure 7 represents only the last two dimensions. The

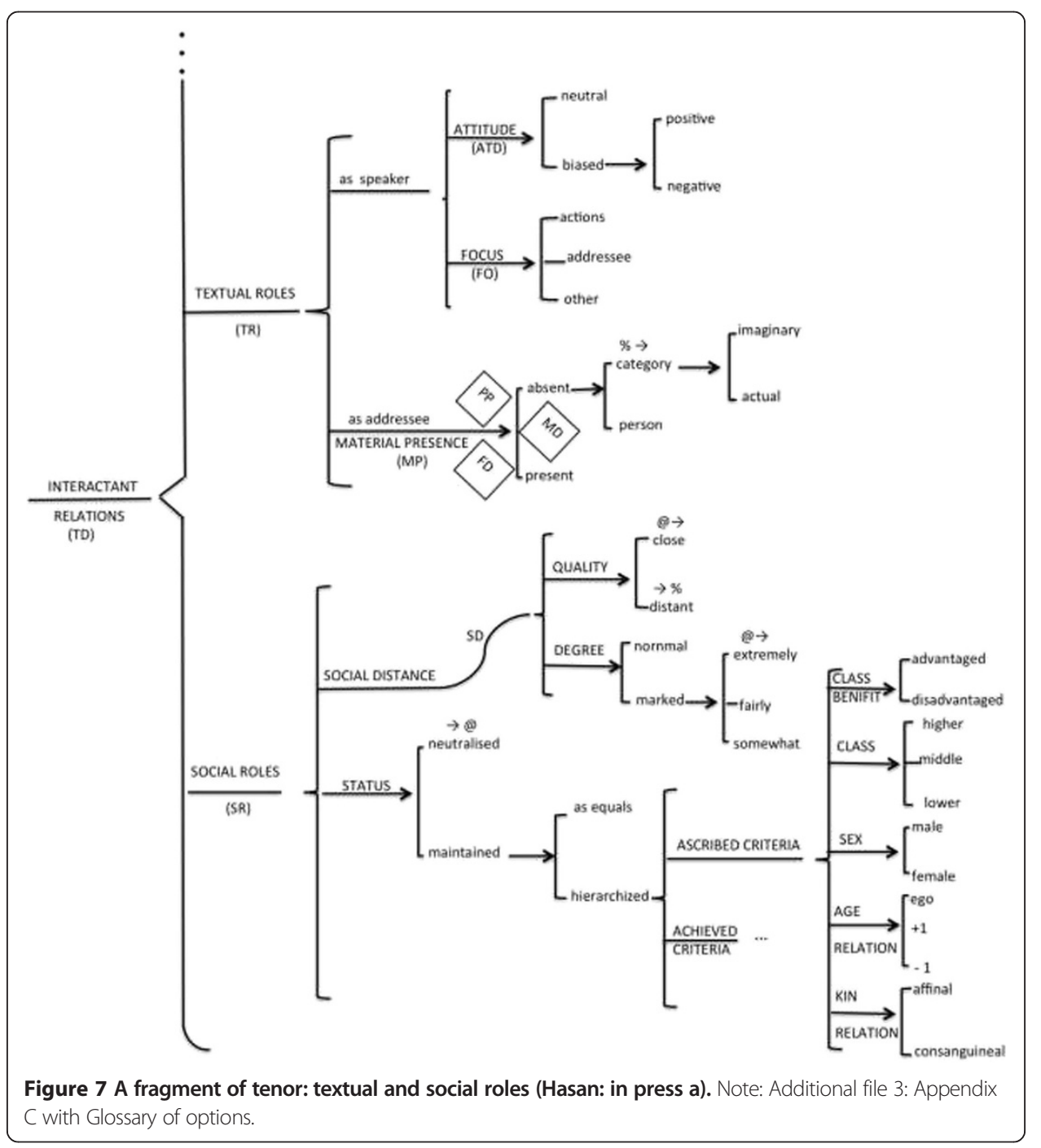


systems of TEXTUAL RELATIONS are naturally pivotal to register identities: since there is no text without a speaker, the speaker is always 'there' both as a material presence in the material situational setting and as a semantic presence inscribed in the text. The primary systems of the TEXTUAL RELATIONS form a simultaneous set. The first $\mathrm{m}$-system here pertains to the interactant AS SPEAKER and opens with a simultaneous set with two member systems, speaker's ATTITUDE and speaker's FOCUS. The second primary system of TEXTUAL RELATIONS concerns the interactant AS ADDRESSEE; this is a simple individual system: the MATERIAL PRESENCE (MP) of the addressee is central from the point of view of text production; not surprisingly, this system attracts many diacritics - which is to say its effect permeates both field and mode. The figure displays two distinct kinds of diacritic: (i) the diamond diacritic, and (ii) the arrow diacritic. Both pertain to the same stratum but the diamond diacritic is inter-systemic and permeates the other two parameters, whereas the arrow diacritic is local, i.e., the relations in one m-system affect those in the other(s) (Hasan, in press b).

The use of the diamond to indicate an inter-systemic relation is part of the early history of the sys-net in SFL: for example, in presenting 'English system networks' where, most probably, this notation was used for the first time in written form, Kress (1976: 101) says: 'This paper, previously unpublished, was written between May and August 1964 and formed the substance of a course on the description of English given by Halliday in Indiana University during that time'. Its use has, however, not been much in vogue since the 1970s. I hope to show that the diamond still has its uses. In Figure 7, three diamonds jostle for space around the entry indicator for the system ADDRESSEE'S MATERIAL PRESENCE (MP). Their attachment to this system indicates some relation between the host system here and those systems whose address is shown in the diamonds. Those addresses take one to both the MODE OF DISCOURSE (MD) and the FIELD OF DISCOURSE (FD), as well as indicating by the third diamond that the choice of 'present' in MP is specifically relevant to the choice of options at the PRODUCTION POINT (PP) in MATERIAL CONTACT. The latter were briefly discussed in the previous section by way of the complex entry point.

Two interrelated problems inhere in this mode of identifying diacritics. First, the identity of a sys-net - where it begins or ends - is not fixed in absolute terms: it changes with changing perspective. But it can perhaps be stipulated that every system that describes any aspect of one particular point of origin, from one metafunctional orientation, is equal to 'the same sys-net'. Even if the earlier concept of 'comprehensive system' (section The technique of the system network: some basic concepts) is jettisoned by limiting it in this way, it may still not be possible to visually indicate all of the relations across two or more sysnets by drawing connecting lines. Related to this is the second problem: whatever method of connecting parts of the sys-nets related by permeability, some reliable way of identifying them has to be found such as the addresses MD, FD, PP and so on in Figure 7. Naturally this will work efficiently only if it can be decoded correctly. One way of ensuring this is to 'standardize' terminology as for example with terms such as 'verb', 'noun', 'word', 'phrase' etc. (although still ill-defined). The suggestion is that just as a language of description has been created to refer to lexicogrammatical category, so also a standardised language of description must be introduced to refer to the contextual and semantic categories. As in each such inter-systemic connection, at least two distinct sys- 
nets will be involved, there should be a convention of cross-reference: the marking in Figure 7 should be reflected explicitly in Figure 6, by showing at PP the relevance of MP (MATERIAL PRESENCE of the ADDRESSEE) as relevant to the choices in MODE OF DISCOURSE. But of course the primary issue is to decide whether it is better to describe addressee's presence/absence as a feature based in interactant relations or whether there is some better argument which favours the placing of these features under material contact in MODE OF DISCOURSE. My reason for favouring the proposed diacritic is that the presence/absence of addressee is relevant to choices in other m-systems of tenor: the appropriate place for options pertaining to addressee seems to be in tenor which is about the relations of speaker-addressee roles.

I move now from the inter-systemic relation, to the 'intra-systemic' one, which is signalled by an arrow. The first clear gloss I find for it is as follows (Halliday 1973: 47):

$$
\mathrm{a}^{* \rightarrow} \ldots . \mathrm{x} \rightarrow * \text { [or any paired symbol] } x \text { is unmarked with respect to } a \text { [if } a \text { then } x \text { ]. }
$$

As shown above the relation is signalled not by one arrow but by a pair of arrows. In fact the arrow signal has three elements to it. First to occur is some symbol e.g. \% attached to that particular option to which the relation applies. If that symbol is located before an arrow as in $\% \rightarrow$ this constitutes the 'if clause' (to be read here as: 'if in MP the option 'category' is chosen'); then if the same symbol follows the arrow (e.g., $\rightarrow \%$ ) and is attached to another option in the system, this would act as the 'then clause' (to be read here as: 'then in the system of QUALITY, only the option 'distant' can be chosen). This clearly shows that the relation indicated by the arrow diacritic is 'preemptive': given the specified circumstance, the privilege of free choice is pre-empted from the starting option, which must combine with the option indicated by the second pair part of the diacritic (and whatever other relations that might entail).

The first of these intra-systemic relations occurs in Figure 7 where the starting arrow marks the option 'category' in the system of MP, marking the IF condition $(\% \rightarrow)$; the second pair part, marking the then clause $(\rightarrow \%)$ on the option 'distant'. So according to Figure 7 choosing the option 'category' in MP system pre-empts other choices than that of 'distant' from the system of QUALITY, the first member system of the simultaneous set deriving from SOCIAL DISTANCE (SD) which refers to a kind of SOCIAL RELATION that arises from the interactant's interactive biography. In effect, by using this diacritic what the sys-net has indicated is that IF the speaker is interacting with a 'category' of addressees not known personally to the speaker, THEN their social relation arising from their interactive biography can only be 'distant'. However entering into that system of QUALITY in SOCIAL DISTANCE entails also choosing from the m-system of DEGREE; the choice from here will depend on the nature of the options permitted by 'category'. If the category is 'imaginary' - i.e., the speaker has not personally encountered any specimens of that category - then the degree of personal relation is likely to be 'extremely' ('distant'); but if 'category' is more delicately described as 'actual' - meaning that the speaker has actually met some specimens of that category, e.g., the lecturer in relation to the first year class - then the degree of personal relation might vary accordingly: what cannot be changed now is the choice 'distant $\mathrm{t}^{\mathrm{r}}$. This follows from the fact that a realisational statement once made cannot be abrogated (for more detail, see section Realisation: the renewal of connection with experience below). In this same system of QUALITY there is another signal for a pre-emptive choice relation; it is attached to the option 'close' and also 
to 'extremely'; the arrow diacritic that signals it, appears as @ $\rightarrow$ whose second pair part is attached to the option 'neutralised' in the system of STATUS (c.f., SOCIAL RELATIONS = SR). Here there is a complex 'IF clause' - IF 'close' and IF 'extremely', THEN in the system of STATUS, all choices are pre-empted; only one option, i.e.,'neutralised' is allowed.

The relations indicated by the above diacritics will be found also in sys-nets representing the description of semantic or lexicogrammatical units, though perhaps both kinds of marking seem to occur more densely in the systems of context than elsewhere. That said, without further research, I am not able to say exactly how this actual fact might be interpreted; two explanations come to mind: either less attention has been paid to the facility of diacritics in doing semantics and lexicogrammar or the contextual systems are truly far more permeable than the language internal ones. I am not aware of any full length manual or discussion of what I am calling the representational technology of sysnet; nor am I aware of any courses designed to teach this aspect. So neglect of some facilities seems not an unlikely explanation. At the same time, Halliday has often drawn attention to the fact that language is 'big'; a division of labour has been set up to help with the complex task of description by creating different strata, different units at each language internal stratum, and a clear specification of the relations across strata and between units. Culture cannot conceivably be less big than language, but so far, at least in linguistics, it appears to have been conceptualised as mono-stratal without any clear principles for establishing units: all the relations that have been proposed have remained 'informal', i.e., no recognition criteria have been set up. The only organisation imposed on it is that which arises from its instantiation by contexts and its resonance with metafunctions with their foundations in the essential nature of human social practice. Seen from this point of view 'The formal properties of any given language event will be those associated with the interaction of field, mode and style' (Halliday et al., 1964: 93) so contextual permeability would not be surprising.

Whatever the case, to me the permeability of the parameters has always appealed as a robust feature visible in the details of 'social practice' (c.f., Hasan 1973): it cannot be written off, and I have welcomed the use of both types of diacritics to indicate the 'porous' nature of the contextual parameters. Apart from the relevance of permeability to the classification of registers - are they a help or a hindrance? We do not know because, sadly strictly speaking, this classification has not even begun yet - though permeability does seem to make contribution to linguistics in general. Indication is that ignoring this feature might not help the descriptive enterprise.

\section{The semantics of paradigmatic description}

The foregoing discourse has attempted to establish the status of the sys-net as an efficient device for recording data of paradigmatic analysis. Entered according to the conventions of the sys-net, such input can be reproduced precisely by any one familiar with the conventions. But sys-net cannot check the accuracy of the coded data; so the information it presents is not necessarily valid as description. The pertinence and validity of the entered data is governed by the general theoretical principles for description. Here two relevant reminders: first, the conceptualisation of the point of origin, and, second, a clear understanding of the critical properties of paradigmatic description. The point of origin discussed above (section The technique of the system network: some basic concepts) is the object of enquiry, i.e., the descriptum; this is the concern of the ensuing sys-net; and the process of the paradigmatic analysis 
is implicit in the principles of the theory (for discussion see Matthiessen, in press). Briefly, the aim of this analysis is to describe the potential of the category, and this is achieved by accounting for all those systemic sign-relations that underlie the potential of the descriptum: in fact, the potential of any category is represented by a complete set of systemically calibrated sign-relations pertaining to the category. The analysis of these relations proceeds top down, and with each successive move, it leads to greater delicacy in an orderly way.

Though originally designed for the language-internal categories, these goals and aspirations, in general terms, can apply to context of situation as well (Firth 1957: 181): since the contextual parameters are viewed from the perspective of meaning-wording, their paradigmatic description can easily employ the same principles as used successfully in the description of linguistic units (for relevant discussion, see section Complexity in systemic relations: simultaneous systems). But one problem must be brought to attention: there is just as much emotional investment in context as there is in language, and this is coupled with another fact. Language users, using language for the living of life, know everything they need to about the process of language but hardly anything about language as system; similarly, members of a community know the contexts of living from their personal experience of living but hardly anything about culture as system. The process based knowledge of language and culture is sometimes celebrated as 'native intuition', but 'intuition' used in this sense is simply another name for the distilled experience of saying and doing as a social being. The description of a systemic category's potential, whether linguistic or cultural contextual, does not undervalue such intuitions; but it seeks to extend the description to the complete range of regular patterns of possibilities. The focus of a systemic and functional description has to be on patterns of regularity as found in a community: it is the regularity of communal action, interaction and reflection that forms the foundation of our cultural history. This is the perspective adopted here in describing the content of contextual parameters. Ignoring many details, the discussion below will concern just three essential issues: (1) defining the domain of description; (2) analysing the analysed categories; and (3) validating the description by using the realisational relations.

\section{Defining the descriptive domain}

Treating the point of origin as the ultimate descriptum implies that the understanding of its nature is the first step in the descriptive enterprise. It is expected that the process of description will reveal details about the descriptum not suspected before the actual analysis began. In the case of context, the familiarity with the words 'context' and 'situation' has perhaps been rather deceptive: the first to raise an alarm against this tendency was of course Firth in his critique of Malinowski (Firth, 1968). But, even today it appears necessary to stand back and ask two basic questions: the first concerns the relations of culture and situation to language as the object of enquiry in the science of linguistics, and the second concerns the positioning of context in systemic functional linguistic theory.

Section System networks as a language of description with its sub-sections leaves no doubt that the description presented in a contextual sys-net is an 'un-packing' of simply some aspect of these terms. This unpacking cannot happen in one fell swoop, but option by option, system by system, and SE by SE the descriptions represented in the sys-nets build a profile of what contextual parameters stand for. So, in the linguist's 
concept of the point of origin will lie the virtual beginning and end of the description: this is where the options will come from; this is where their relations will be rooted, which is precisely what description will be attempting to represent. There is no book of rules about this process, but we luckily do have some wise advice from Firth: the interpretation of terms such as context of situation is best approached theoretically. That can only mean one thing - to understand how this concept is related to others in the theory, and how they act together to achieve the goals of the theory.

From this perspective, the CMR hypothesis appears attractive. The hypothesis presents the most comprehensive and theoretically based proposition that linguistics has had to offer by way of defining the domain of the three parameters of context. With reference to context and meaning-wording, it recommends retaining the integrity of each contextual parameter by treating each as metafunctionally oriented, and, in so doing, it offers general criteria, for recognising the contextual features and their linguistic realisation: the correlates in register variation are subjected to a principle. So far as the metafunctional hypothesis itself is concerned, it has been used successfully in SFL, as is evident from its use in the description of both semantics and lexicogrammar. If the lexicogrammatical descriptions and their meanings (Halliday and Matthiessen 1999; Hasan 1983; Cloran 2004; Williams 1995) have proved reasonable, then the use of this approach is worthy of a serious trial. This makes register classification a rational and feasible project. The paradigmatic mode of description and the technique of its representation as in a sys-net are well suited to testing the probabilistic hypothesis concerning the realisational relations of situational and linguistic features. It is not necessary to repeat that the probabilistic nature of the hypothesis can be handled satisfactorily. If using the full range of representational facilities, the analysis of contextual parameters reveals a significantly higher degree of cross referencing in and across the sys-nets than considered valid in terms of the default metafunctional orientation, then the CMR hypothesis will clearly need to be revisited; it may even have to be jettisoned if a better hypothesis is available, though indications are this would not be the case, c.f., Figure 7 where the markings are not at all dense compared to the number of options that appear not to require cross reference.

\section{Analysing the categories of analysis}

In SFL description works its way from top down: more specifically, it begins with a category and proceeds by identifying significant properties by reference to which that category may be validly sub-classified; and as the next step, each of the latter properties is further analysed to allow more delicate sub-classification, continuing in this manner right up to the most delicate properties. Thus the first step in this process of description is the conceptualisation of the top-most category: it is the only un-analysed term in the entire comprehensive sys-net, known as the point of origin; it is the analysis of this term that gives entry to the primary system with at least two options; and following the above mode, the analysis continues what was earlier described (The technique of the system network: some basic concepts) as the 'cycle of birth and rebirth' responsible for the derivation of the system network. The possibilities of variation in this general process were described above. Based on the implications of this condensed account of systemic descriptions, some important characteristics of systemic options are presented below. 
A system comes into being only when the possibility of choosing options is available; this makes option and choice central concepts in all system-based description. Clearly the options presented in the sys-net are witness to the linguist's conception of the descriptum; it is difficult to say any more on how options find their way into sys-nets, but some general properties of options may be stated. First, every option is produced in analysing a given category; this includes the primary system, and each next step adds to the degree of descriptive delicacy. There is no logical end to this process: descriptions are in principle open to greater delicacy. Second, the relation of all the options following the 'entry point' of a system typically stays stable; and through the relations of one option to those along the legitimate systemic paths, all the options are eventually linked to the ultimate descriptum named by the point of origin. Third, at any one point in the descriptive process, the properties of each chosen option are described exhaustively by all those options which will constitute the next step; it is assumed that, according to the analyst, no more can be said at that given degree of delicacy about that previously chosen option. Fourth, each option of the system(s) at any given point labels a distinct property of the term whose analysis brings it into being; each further step in delicacy introduces new properties into that analysis. Fifth, each system, whether individual or an m-system, has a particular perspective: often, overt labels are not attached to each system in the network so as to avoid crowding the space for representation, but when a label does appear it is expected to conform with that perspective. In other words, options named in the system will be in 'keeping' with that system's perspective; to give a trite but clear example, no competent linguist would create a simple system that displays the options 'narrate' or 'phonic'. Sixth, if there are simultaneous sets, this requires a free combination of options across its m-systems; in that case, either all options will be compatible, or in order to prevent internal contradiction, an arrow diacritic will indicate some pre-emptive moves. Finally, each sys-net having covered some distance will produce some selection expressions (SEs), which, by definition, present the relations of the included options. The legitimacy of the SE cannot even begin to be checked in any real sense unless its options display a coherent picture concerning that which they are describing. Since options are presented and ordered in a sys-net with the purpose of displaying the description of a category, both their compatibility within the SE and their applicability to what is being described are necessary conditions for their acceptance. No matter how huge the sys-net, all its options are mutually related: this interconnectivity is what underlies the concept of a comprehensive sys-net.

The above is then a general statement of where the options come from and what they are capable of doing by way of description. Obviously the clearer the ideas about what constitutes the domain of description, the more consistent the description is likely to be. The sys-net itself has no concern with the semantic notions of consistency, compatibility, appliability and so on: so long as it is 'readable', i.e., the signals are valid as systemic conventions. The sys-net presents what has been put into it as the offered description. Validity is a semantic notion; it does not concern the sys-net conventions per se: it is the analyst's concern. If it is accepted that the 'cycle of birth and re-birth' of options in systems is what underlies sys-net derivation and that sub-categorisation is what underlies the descriptive continuity, then obviously an understanding of what the entry point means will be relevant to the selection of the options and to the relations between them; changing mid-stream the conception of what the system's entry 
point means is likely to create confusion. For example, if the dimension of FIELD OF DISCOURSE is conceptualised as concerning activity, then the systems introduced by its entry points could cover everything that classifies the nature of action, such as types of action ('material'/'verbal'), the sphere of action ('local',/'institutionalised'), and the status of action ('quotidian'/'specialised'). Obviously not any one can engage in every activity: class, expertise, maturity, sex, and prestige of the doers come readily to mind as some constraining variables. Should these vectors be therefore brought into field? This depends: if the description is purely 'notional' then nothing stops the analyst; otherwise tenor is where these properties of interactants would seem more appropriate, and very strong reasons are needed to move it from that parameter to that of mode or field. The price to be paid would be a cross referencing diacritic; but the advantages from following the practice appear considerable. Apart from the fact that it maintains the CMR predictions thus avoiding regressive consequences, it is very likely that these vectors would be relevant also to choices in the system of STATUS especially in maintaining HIERARCHIZED ROLES. And whether or not this will apply could very much depend on the choice in SOCIAL DISTANCE: if the options 'close' and 'extremely' apply, then hierarchical constraints might be 'neutralised'.

Inside the system there is no time or space; given the acceptance of greater delicacy as the method for exploring the limits of some potential, the issue is simply what relations need to be selected, what consequences the selection of one option in a system has for another; how the meaning of one relates to that of the others. So before and after, previous and following are used with a meaning that may be quite different from that in which they are applied to material time and space. In the absence of material time and space, what guides the logic of placing a system of options here or there rests on ideas about what would lead to an exhaustive, accurate and explanatory description of that which is being described - and that is implicit in the analyst's view of the theory. Thus, here, mode of discourse is conceptualised as about ways of establishing contact between the speaker and the addressee: so when a simultaneous set of primary systems is presented (as in Figure 6) with the first system describing the nature of the MATERIAL CONTACT, the implication is that all phenomena describable as that part of MODE i.e., ways of creating material contact between the speaker's message and the addressee can be exhaustively described by using the options of systems included in that system. If on re-examination the network appears lacking, it may still be retained in so far as it is satisfactory, with necessary modifications added or and developed. If, however, there is contradiction and/or other issues that cannot b resolved, it might become necessary to go back to the drawing board and create a totally new sys-net.

One quick comment on context as material phenomena. According to one common saying, seeing is believing: the immediacy of experienced reality is of course undeniable. It seems to me that acknowledging the truth of this axiom does not entail ignoring the fact that reality is, to a large extent, refracted through language. Freed from the limits of vision and sensation, the scope of this reality is beyond measure; it is a reality that nurtures and is nurtured by human minds; it is made of meanings that only the semiological system of language can produce, and that can only be 'read' by minds free of brain pathology and familiar with the functional varieties of that language. This mind, made by the experience of languaging in the community, knows more about the context of situation than the material situation itself can ever show: and the only way we can establish what that mind 
views as 'the context' is to be aware of what goes on in language. Register is after all a linguistic category, and the contextual features relevant to its classification are likely to be those realised as some linguistic patterns. Common sense may declare that no action is without a goal; and goal orientation may appear a relevant situational variable to introduce into the description of field. Indeed this is what I had thought when introducing options such as 'visible' or 'invisible' goal orientation, closely related to another kind of goal orientation, i.e., 'short term' or 'long term's the context for this was a debate in sociosemantic variation in mother-child dialogues (Hasan, 2009b: 93) from the perspective of the role of every day talk in the formation of consciousness, which is an important issue from the perspective of 'socialisation'. Locally, i.e., for that particular set of problems, it had proved helpful. It is true that the semantic style of linguistic action in the long run enacts interpersonal relations: people living together and habitually interacting do tend to develop reciprocal forms of behaviour and attitudes. But in less than a decade I was walking away from the inclusion of these as contextual options; and I did present what seemed fairly careful arguments against my earlier views (Hasan 1999: 233-237): but unfortunately those concepts have been adopted (sometimes under different names), usually with acknowledgment to those detailed reservations but without offering any serious reason for ignoring them. It appears to me now that the entire discourse of purpose, goal, and outcome is fraught with problems similar to any discourse of intentionality in relation to any verbal interaction. Had I paid more careful attention to the writings of Wittgenstein, Firth, Whorf, Vygotsky, Halliday or Bernstein, I might have avoided being guilty of introducing these terms. This is not to say that human actions are goal-less: goal inheres in the action. There are, in fact, no means of knowing which of the invisible goals is being pursued; and from this perspective, it is worthwhile reflecting on the difference between deception that is successful and deception that gives itself away.

\section{Realisation: the renewal of connection with experience}

The centrality of the realisational relation for the semantics of paradigmatic description is quite obvious from the previous pages. A contextual sys-net without an indication of how its options are realised linguistically has serious disadvantages: it is open to misreading; it lacks linguistic validation; and its use in the classification of register is therefore open to doubt. As elsewhere in contextual matters, the discussion is potentially huge. In this section I will limit myself to four issues (i) the function of realisation; (ii) realisation statements; (iii) selection expression; and (iv) the trinocular perspective.

The term 'realisation' was introduced in SFL in the second half of the 1960s to model the relations across the strata. Effortless as it seems, language use (aka parole) is a complex activity, and the model postulates different orders of abstraction, each recognised in the theory as a distinct stratum: what realisation does is to show how one kind of abstraction, say 'semantic energy', may be 'exchanged' for another, namely 'lexicogrammatical energy', which is itself 'transduced' into yet another order of abstraction, namely 'phonology', and that is 'transformed' into actual sound stream (the phonic relay) which is what impinge on the addressee's senses. It is usually difficult to produce efficient synonyms, and more so in the case of theoretical terms, which inhabit a virtual universe. I have settled more recently on the term 'transducer' and its 
derivatives, as the nearest approximation to what 'realisation' does (Lamb, 1966). The first edition of Macquarie Dictionary defines 'transducer' (p: 1834) as 'any device which receives energy from one medium or transmission system, and supplies related energy to another medium or transmission system' [Emphasis added]. So the relation of 'realisation' acts as a transducer, bringing together by a theoretical artifice the different transmission systems that had, in the first place, been separated by an act of analysis. It is not necessary to remind that in using metaphors such as 'transmission', 'translation', or 'transduction', there is no implication whatsoever that in actual language use users will apprehend a distinct transitional point from one stratum to the next: as I myself have said, the process presents itself as a 'seamless flow', in which for the layman the phonic flow 'becomes' the 'flow of meaning'.

This seemingly simple 'fact' of 'perceiving' the binary nature of the process - sound and meaning - has been recognised since the very beginning of deliberations on language. However, as Saussure himself pointed out this too is, in fact, not a fact, since if meaning and sound stood apart, there would neither be a signified nor a signifier; in this sense their perception as a beginning and an end is also a fiction. In terms of Saussure, the inherent make-up of language is 'irrational'. Be that as it may, no functional linguist would explain meaning in terms of phones; the solidarity of meaning is on the one hand with context, which acts as the measure of the relevance of speech, and on the other with wording, which is the construer of meaning. Semantic and lexicogrammatical units are abstract; they can make no direct connection with the human body; and to be accessed by the intellect there has to exist some association which renders the bodily accessed phonic/graphic pattern 'intellig-ible'. Semantics has been described in SFL as an 'interface'; but its relation to material situation is probably no more direct than that of phonetics to air waves. Modern linguistics has been and still is searching for an explanatory account of how this web of myriads of specific associations between the sens-ible and the intellig-ible gains currency so as to enable all the varieties of linguistic interaction, known as registers.

Different ways of notating realisation have been suggested in SFL (see Hasan 1987 for some discussion on kinds of realisation statement). A rather arbitrary demand was to place the realisation of a particular option close to where that option occurs in the sys-net. This, like the demand to show the name of each simple system in a sys-net, becomes impracticable when, over the years, the description plumbs the depth of complexity in the category, which leads to a sys-net thickly populated with systems and options. The alternative is to tabulate the realisations, option by option, as presented here in Appendices A - C, or as a table/note below the sys-net itself, as illustrated in Figures 2 and 3 in this paper. Ideally the realisation statement should be made in terms of the systemic features representing the categories of the stratum below: so the contextual systemic features are shown to be realised as semantic, the semantic features as lexicogrammatical, the lexicogrammatical features as phonological, and the phonological features as phonetic ones. In this way the two strata primarily concerned with substance are woven into language, since neither context nor phonetics is a picture of 'what is really out there': context is conditioned by its relation to semantic patterns, and phonetics by its relation to phonological patterns. This way of presenting realisation statements has been tried to some extent in systemic functional semantic studies (Cloran, 1994; 1995; 1999; Hasan, 1983; 2009b; 2013; Williams, 1995). 
However, much of the description, especially on the stratum of semantics, remains rather 'patchy': while it is comparatively easy to offer the semantic features relevant to the textual option 'present' (Figure 7) it is far more difficult to do this for a term such as 'imaginary' as located in the choice path 'absent: category: imaginary'. So, often, a realisational statement of contextual feature is made informally by offering a gloss, as in Hasan (1999; 2009b or appendices/tables here) and Butt (2004; and Figure 2 here). The progress in lexicogrammar raises hopes that this problem may be overcome in time (witness, for example, mood sys-net in Cloran, 1994 compared with mood in Halliday and Matthiessen in 2014). A far more serious point is that the meanings of terms referring to options in a particular sys-net are typically specific to that system: their meaning is completely conditioned by 'the company they keep'. 'Unifying primary options' from distinct systems is to lose the specific meanings of those options in the original sys-net.

This is where the concept of 'selection expression' (SE) comes in as a device that preserves the original meanings. Referred to earlier, SE presents a complete statement of all the options and their relations that are traversed in one single systemic path of a sys-net from its primary delicacy to the terminal point; as such, it represents the complete 'history' of that systemic path: underlying each SE is a contextual subcategory, whose realisations would pertain to some text type. In order to preserve an accurate history, every realisation statement is permanent: nothing in it can be altered, deleted, contradicted or inserted (except as a form of increase in delicacy). I believe this is what Mann (1985) had in mind in introducing the concept of REALISATIONAL INHERITANCE: within the constraints of a specific systemic path, the relational statement of every preceding option will be respected by the options related to it by derivation. It is important to emphasize that each option has a unique though related significance: in the end, all options pertain to the ultimate descriptum, but each makes a unique contribution to the profile of some underlying category. For example, if the primary option 'phonic' is glossed as 'relay at PP as sound stream/ phonetic', the meaning of this statement cannot be altered at any point in that sys-net. These principles are clearly necessary to ensure the validity of the description. Continuing with the example of 'phonic': if a choice path represents the relation 'phonic; direct: congruent' this, though true, is redundant information, since 'phonic' will always inherit 'relay at PP as sound stream', so its natural form would be oral and its 'direct' reception would therefore be aural - it cannot be anything else than congruent. Thus the option 'congruent' fails to add new information; so it is not a 'good option'. By contrast, 'phonic' in conjunction with 'mediated: congruent' tells a significantly different story - that despite the intervention of some agency, human or mechanical, the 'natural' pairing of oral-aural is maintained; moreover a credible variant possibility exists that if 'mediated' reception could have been 'incongruent'; the 'spoken' may be received as writing. So the placing of the options 'congruent' or 'incongruent' as in Figure 6 is informative: the choice path 'phonic; mediated: incongruent' may be glossed as 'original relay in speaker's voice mediated mechanically is accessed by some other modality'. Previously in presenting this analysis in lectures, I had adopted the expression 'spoken as if written'. The present version appears preferable, as it does not confuse channel features with the style of wording. Whether the description is 'correct/valid' or not will depend on the 'renewal of connection with data' as Firth suggested: when it comes to language, the validity condition depends on whether the community recognises that convention for 
presenting meaning-wording-expression? If the answer is 'yes' then instantiation and realisation meet in the instance of the system, i.e., the text. There is a theoretically significant difference between 'instance' and the actual happening. The actual may depart from the 'typical' in unpredictable ways. With this emphasis on the 'typical', the value of a sys-net may be described as determined by the degree of its validity.

To conclude this discussion of SE, it is not the number of systems in a sys-net or the options in the sys-net that make it 'good': what matters is how accurate and insightful the description proves to be. And this depends on two characteristics: one, the validity of its description - what categories of registers it can identify that would be communally recognised as language varieties, and secondly, how accurate its statement of the relations of options as represented in the sys-net are. If either is open to doubt, no matter how 'extensive' the set of systemic representations, its value is questionable. The SEs of a sysnet present a 'summary' form of the many systemic paths created by the relations of dependency and simultaneity between the options. Unless the paths themselves are wellformed, the question of validity cannot be reasonably raised. So if we take Butt's contextual description of the FIELD OF DISCOURSE, this opens with a simultaneous set consisting of four member systems (2004: 24). This means that, unless otherwise indicated, the options of each of these m-systems are combinable within the limits placed by mutually exclusive options; the computed SEs in this description will be so enormous that their calculation or representation is beyond my means. But take just one system, that of GOAL ORIENTATION (Butt, 2004: 35), which itself opens with a simultaneous set consisting of three members; and again as before no constraint on the free combination of the options is indicated. But the combinations do appear quite problematic: for example, option 'immediate' ${ }^{1}$, when combined with 'unconscious ${ }^{4 \text { ': }}$ inaccessible ${ }^{4.2}$ ' makes little sense. The option 'immediate' ${ }^{1}$ ' is glossed as 'Game-Win or Auction-Buy'; but it is not clear that it is goal orientation that is 'unconscious: inaccessible'; how can one have an orientation to Goal without knowing what the goal might be. Similarly, the combination of the three terms referred to Hasan (1999), i.e., 'independent' or 'aligned' or 'integrated', with the option 'immediate' or with 'longitudinal' would be quite problematic. It is worth pointing out that at present hardly any contextual sys-net is more than tentative: all are at a nascent stage. The danger in taking the 'extensive' sys-nets at face value is that with so many options in the sys-net something from the array of options could be applied to an actual case as being 'the best amongst those available'. In the absence of even a few indicative SEs, it is not easy to know what one is doing and why.

In this situation, to gain some idea of the complexity of the realisational relations, it may be helpful to turn to the concept of 'trinocularity' (Halliday 2009: 79-80). Known as the 'trinocular perspective', it can be adopted in the investigation of any category from three closely related points of view: the perspective can be applied in the environment of other axes, but my concern here is with realisation relations, particularly with reference to text-in-context, and I shall focus on the three contiguous strata on the 'content plane', namely context, semantics, and lexicogrammar, since these are closely relevant to the production of the meaning-wording that constitutes the semantic face of text. Even though the spectrum of the entire verbal interaction will not be described, trinocularity can provide a good insight into the intimate realisational relations from the perspective of these three strata. Stating a general principle, in the middle of the trio is some category that realises some feature(s) from 'above', and is itself realised by some feature from 'below'; while 
'around' the middle category are the relation of the category under focus to those others in its family that are instrumental in defining its identity.

I begin with a sketchy scenario in order to demonstrate the trinocular perspective in action: suppose that the middle stratum is the semantic one, then the stratum 'above' is context, and the one essential material element is a human being in relation with another: the roles they bear is that of 'speaker/addressee'. I have argued that the speaker role is always materially present in the situation and semantically present in the text, i.e., in its relevant context via meaning-wording; as opposed to this the addressee role is always semantically present - i.e., the addressee's profile can be traced through the disposition of the text, even when materially absent (Hasan, in press a). Suppose on this basis, the speaker is an employer; his addressee is his secretary; and the social practice is that of planning a response to a message from a third party. The question on the floor is when this activity should be undertaken; and in response to this query the speaker will need to use some variant of 'demand; service' (Hasan, 2009b; 2013).

Now, in terms of contextual features, the kind of field they are concerned with is managing the performance of a category of 'doing', such as despatch, send, write, respond, and a category of related objects, e.g., response, reply, message, letter. This observation presents a profile of field where action has the features 'verbal: constitutive'; sphere is 'specialised' (as in Hasan 1999: 311). In producing the meaning-wording, i.e., the text, the speaker/addressee will refer to at least the category of actions and objects just noted as relevant to the context. The scenario implies that the textual role of addressee is materially 'present'; this suggests the relay at point of production will be 'phonic'; since the exchange between interactants is face to face. Since the addressee is materially present, and the activity is negotiated planning, the textual roles are potentially 'reversible'. Their agentive role is 'quasi permanent'; that is to say, the likelihood is strong that they regularly interact in a specifiable capacity (here postulated as employer-employee). The social distance between them is very likely to be at least 'normal' (that is, in keeping with the employeremployee relation) or 'somewhat; close'. Whichever the case, it implies that the textual roles are more likely to be 'reversible' than not: the secretary is not simply listening; she is contributing to the plan on the floor. This might mean that the feature 'spoken' in the system of ELOCUTION in SEMANTIC CONTACT (SC) will combine with the options 'phonic' 'direct' from the system of MATERIAL CONTACT. In view of the text's context and the interactant relations stated so far, 'phoric' reference to previously mentioned actions, objects etc. can be expected by way of CONTINUITY in SC. This is a brief and indicative account of what the interactants are extremely likely to 'know' about their interactive context of situation in which they will take an active part. There is certainly more to the information provided contextually, but hopefully the above account will suffice in indicating how the contextual features activate the meaning-wording produced by speaker/addressee as their text. Remarkably, as contextual features and the meanings that realise them are discussed, they would appear to support the predictions in the CMR hypothesis.

Turning now to look 'around' at the stratum of semantics: here the question is what resources are around for demanding goods-\&-services, i.e., for choosing, as a response to the secretary's question on the floor, some appropriate pattern from the family of messages that consist of 'getting someone to do something' (Hasan, 2009b; 2013). The semantic stratum can present a large set of possibilities each of which will be heard as 
some kind of demand to undertake an action in a particular way so as to make a difference to the existing scenario, varying in what goods- $\&$-service are being demanded, and what relation the asking will 'enact' with the addressee. It is highly probable, in view of the interactants' tenor relations as described above, that the command will NOT have the feature 'plea' or such 'softeners' as 'if you don't mind', 'if it isn't inconvenient'; and it is even less likely to be 'suggestive' e.g., 'lets do it straight away, shall we?' But even counting out these categories, the speaker might take an assertive stance as in 'I would like it done straight away' or 'it better be done straight away'. A more authoritarian near mandatory command might be 'you had better/you should/ you must do it straight away'. Compare this with 'it should/ it must be done straight away'. And all of these can be compared with consultative commands 'can, will, could, would, won't, can't you do it...'. As well, there are a large number of exhortative commands such as 'do it..., you do it..., better do it...'; and in this context consider the quality of relation enacted by the selection of such additions as '... will you?, ... can you?, ...would you?, ... could you? ... can't you?, ... won't you?'. Of course, more details about the selectable meanings can be added; but perhaps this will suffice to indicate the resources 'around' in making demand for service and the 'construal' of context by meaning-wording. In the end, the employer's response to the question does not depend purely on 'what' the action is, but more likely on his ATTITUDE and FOCUS with regard to the addressee (see Figures 6 and 7 for the terms used with reference to mode and tenor).

But there is also the stratum 'below': no language use can come about without the formal resources of the language, and these lie at the lexicogrammatical stratum in immediate relation to 'meaning in context'. In the functional modelling of language, form does not disappear as sometimes implied in setting a binary choice between form and function; nor need it be notional (if notional means intuitive). Rather, the form is seen from the point of view of its own resources as form, and at the same time the question is always raised 'what difference would it make to activate the option declarative as opposed to interrogative or imperative, tagged as opposed to non-tagged, positive, as opposed to negative, and so on. The separation of strata is a theoretically created 'fiction' and meaning, as used here, refers to 'meanings construed linguistically': it cannot be known without being said. So far as semantics is concerned, the speaker's options can only be known by the wording: what process, what participants, what mood, what modality, what phoric relations, what forms of linguistic interaction - all of this is typically 'put in so many words'. Of course the question of errors and deceptions is important, and if space permitted one could discuss these in light of the function of co-text, and of 'reading to make sense' either by direct reference or implication. What will be seldom found is the surrogate relation of 'correspondence' element by element to some reality out there: the real reality escapes us; the reality with which human communities work is construed reality - and construed largely by language. Of course, there will be gestures, eye contact and perhaps other non-linguistic modes of meaning: but my focus is on what language can do. While revealing the immense complexity of the process of 'meaning making', the concept of the trinocular perspective brings us considerably closer to answering that important question: why does anyone say anything to anyone? In bringing about this outcome, SFL has thrown considerable light not only on how language works, i.e., by what resources, but also on why, i.e., the motivation. The orders of relevance established to answer that 
beguilingly simple question would most probably need to be taken into account in any credible neurocognitive profile of what it means to speak.

\section{Concluding remarks: the value of a scientific study of con/text}

Earlier in this article I have suggested that the paramount need in the description of contextual features is precise and orderly statements whose validity could be judged using theoretical principles. A case was made for testing Halliday's context-metafunction resonance (CMR) hypothesis as the approach most likely to meet those needs. The paradigmatic description of contextual parameters in an earlier section has demonstrated that precision and orderliness are built into the paradigmatic method of representation: there has not arisen any need to add or subtract from those conventions; all that is needed is to observe its principles as a language dedicated to presenting a specific kind of information. I have indicated some grounds for concern in the way that the systemic representation of paradigmatic description is being carried out. In my view, unless reconsidered carefully, this neglect of the principles of a precise methodology is likely to delay the achievement of the goal we have set ourselves. And that goal as I have argued requires criteria for recognising linguistic and contextual correlates. I have also attempted to indicate the need for the careful labour necessary in achieving this goal. One is of course entitled to ask if the study of context deserves the attention and care being demanded here. In modern linguistics until quite recently context had been viewed as an optional extra. But to perform their functions in the life of the community, neither context nor language depend on conscious recognition: Hjelmslev (1961) had remarked that the nature of language is to remain invisible; exactly the same is true of context. Context has not been relevant only in ancient theories of rhetoric; it has also insinuated itself into categories of grammar such as noun and verb, mood and case, number, person, gender and so on. And some might say this unconscious play of context has worked fine for logicians, philosophers, and supposedly for purely formal grammarians. Why do we need to change this centuries old uneasy relationship now?

The reason is quite simple, though it might surprise: a scientific study of language needs to include within its fold not only langue, but also parole, i.e., language use. This conviction is central to SFL, a linguistic theory that in my view has followed (most probably not intentionally) in the steps of Ferdinand de Saussure, without accepting his paradoxical views on langue and parole. Elsewhere I have argued (Hasan 2013; 2014) that Halliday's SFL as it stands today comes nearest to Saussure's dream of the linguistics of langue, but it does so not by following his disjunction between langue and parole but by showing that his goals can be achieved (with due respect to him) only by ignoring his exclusion of parole. The basis for making this claim is very briefly as follows:

1. variation inheres in language (Saussure 1966); activated by context, it is realised in parole;

2. Saussure's linguistics of langue recommended the study of langue in isolation from parole by reference to a single synchronic état de langue;

3. but as Saussure himself recognised, even a synchronic état de langue will have some variation related to age, gender, profession, and social status;

4. the idea of linguistics as representing comprehensively the description of one homogenous synchronic état de langue is a theoretical fiction: it is certainly not 
beyond possibility that to provide a full and explanatory account of what language is like linguistics will need to account for variations of all kind - not simply diachronic but also synchronic;

5. even supposing the above is not the case, which must be seriously doubted: consider 'World Englishes'; these do not differ just in accent - expression - but also in their semantic and lexicogrammatical designs. A series of successive synchronic états de langue will clearly provide evidence of diachronic variation. And on the assumption that diachronic variation does not occur instantaneously as Saussure has himself argued and as historical linguistics has clearly demonstrated, some explanation will be needed for similarities and dissimilarities across the various états de langue.

6. besides being simply a theoretical fiction (c.f., 4) a linguistics of langue sans parole will be unable to account for these phenomena; the price for a pure linguistics of langue is failure to understand the true nature of the semiological system of language;

7. Saussure thought that the foundation of langue is communal; he banished parole claiming that in parole "Execution is always individual, and the individual is always its master' (Saussure, 1966:13). Even though these statements may be true with respect to the expressive acts of utterance, Saussure himself argued cogently that as a meaningful entity the existence of the sign depends on its social currency.

Moreover, parole is not chaotic as he implied; as Halliday (1991/2007) has argued, the separation of langue and parole is based on the mistaken belief that they are two distinct kinds of phenomenon;

8. without an understanding of parole as active in the evolution of society and in the development of biological organisms into individuals, Saussurean linguistics of langue would be unable to defend claims about the relations of langue and parole, including a principled study of synchronic and/or diachronic variation. In fact, the linguistics of langue would, alarmingly, have no basis for explaining which associative bonds create the grammar of a given langue; for example, what is the evidence for saying that there is a bond between 'enseignement' and 'éducation'?

9. a paradigmatic description of con/text, as recommended here, is very likely to reveal the orderly nature of parole: the evidence for the 'morphology of meaning' lies here;

10. his item based 'associative bonds' can clearly not be viewed as 'templates' of any kind; and they could never reach the breadth and depth of the paradigmatic description as produced by SFL in the description of the internal strata of language (this is not to imply either that SFL descriptions are complete, or that they have been validated at every point).

I am not claiming that SFL set out to be a post-Saussurean model: but as Saussure's work (1966; 2006) suggests the aim of linguistics for him is to make statements about the production of meaning in verbal signs by using an objective, methodical and precise analysis based on templates, not instances. So far as the concept of language as a meaning potential is concerned, the paradigmatic analysis of the internal patterns of language as templates has been most highly developed in SFL. In addition to this, the theory has shown that parole, i.,e., language use, can be scientifically described, so that underlying those descriptions are explicitly stated principles. The methodical study of con/text is not part of the enumeration of what there is out there in the physical world; rather, the theory is concerned with that reality which language is able to construe. This 
seems to be a justifiable goal to pursue, for in actual fact it is this linguistically construable reality that constitutes the best evidence of the meaning-making power of language. The contextual parameters are part of that story: as Firth reminded us, contextual categories are theoretical constructs and they bear no direct relation to items of linguistically non-construable realia. In this respect, contextual categories do not differ from grammatical ones. Their value lies is their 'tool power' (Butt 2005), and that tool power is an artefact of their positioning in the theory.

In this perspective, the relation between theory and description is very much like that between system and instance - instance, not as an occurrence in time and space, but as something that is a realisation of some permissible conjunction of descriptive categories: nothing can be an instance of the language system that is not ultimately related realisationally to some categories specified by that system. As suggested above, the foundation of the three contextual parameters lies in the innate human urge for (inter-)action: the conjunction of some range of Action, some kind of Relations and some means of Contact (ARC) is perhaps the first 'intimation of the social world' for the human infant - and it provides the infant the first opportunity for the exercise of that innate faculty for calibrating the signal and the significative possibilities that in some way become associated with that signal. The CMR hypothesis is not just a hypothesis that makes it easier to identify the linguistic and contextual correlates, important though that is: the resonance is there because the origin of metafunctions as well as of the tri-partite makeup of human social practices is in some way inscribed in the regular patterns of human inter-action. Halliday (1991: 2007b: 174;) puts it this way:

As I wrote myself many years ago, language is as it is because of what it does: which means because of what we do with it, in every aspect of our life. So a theory of language in context is not just a theory of how people use language, important though that is. It is a theory about the nature and evolution of the system, explaining why the system works the way it does.

In a linguistic theory whose title has, for decades, carried the words 'systemic functional' these issues cannot be set aside: the way the nature of the con/textual parameters is viewed has a reach that goes beyond local issues to the very foundation of the theory. In this way, I return to some of the same concerns with which I had opened this paper.

Whatever the motivation for unifying field and mode, similar patterns of permeability will also be found across field and tenor, and across tenor and mode, as shown by the above discussion of diamond and arrow diacritics with reference to Figures 6 and 7. If the unification of the parameters is the answer to this problem, that would take us back to the pre-paradigmatic description. Yes, without some form of ready material access to the addressee, the speaker would not use the 'phonic relay' - a mode issue (Figures 6 and 7); but when it comes to the system of ROLE REVERSIBILITY - the effects of which are most clearly manifested in free turn-taking, this depends not just on material presence or absence of the addressee, but also on interactant relations: being copresent is one thing, and the constraint on forms of interaction is another; the latter are always sensitive to social relations. It seems that permeability has always been recognised in SFL: thus Halliday et al. had commented (1964: 93): 
It is as a product of these three dimensions of classification that we can best define and identify register. The criteria are not absolute or independent; they are all variable in delicacy, and the more delicate the classification the more the three will overlap.

In some ways the trinocular analysis of con/textual features has not yet begun; and it seems to me the focus is more on what is going on there in the performance of some particular social practice rather than on classes of register. If the aim is to achieve reliable register classification, then attention to linguistic realisation is necessary - it is a natural part of the paradigmatic descriptions whose power and precision has been emphasized here. And I have attempted to show that the conceptualisation of the parameters is in agreement with the foundational concepts of the SFL theory. I would suggest that local solutions of descriptive problems need to be evaluated in the global context of the SFL theory. This is not to say that the correctness of the theory's architecture is beyond challenge: theories, especially in the domain of human sciences, will change even if only because our state of knowledge changes. The question is whether challenge to the theory is made about the basic goals of the theory. Change for the sake of change, change without reason, is typical of trivial fields of enterprise - concerned with appearances without a care for substance.

In repeatedly emphasizing language as the main concern of linguistics, I do not forget that social practices being performed around us in the 'real' world call for much more than language: this emphasis on language, perhaps, goes against the riding tides of multimodality, In fact sometimes it has seemed as if there is no difference between the various semiological systems, but I do make a clear distinction between the semiotic and the linguistic: language is a specific means of making meaning - a means that is not used by any other semiological system. The aim of linguistics is to provide a frame that tests the potential of language for meaning: although these resources may be and actually have been extended in the service of describing other semiological systems, those semiological systems are distinct from language and each of them does call for a dedicated theory. As for linguistics, I submit that although it is very much more advanced in the description of language than are the other disciplines dealing with other semiological systems, there is much more that remains to be said about language, especially from the perspective of semantics, which concerns meaning made by language. There was in my youth a song claiming something to the effect that it is love that makes the world go round: I have always felt that it is the meaning potential of language that makes the world go round for better or for worse. But then, I am a linguist.

\section{Endnotes}

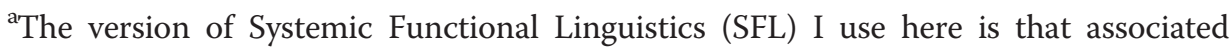
with Halliday's name; I will largely ignore the variant versions (e.g., Martin, 1992 or Fawcett, 2000) as some generalizations made here might not be applicable to them. I thank Michael Halliday for commenting on an earlier draft of this paper. I alone am responsible for the views/interpretations of the theory as presented here.

${ }^{\mathrm{b}}$ As an admirer of Firth, I do not make these remarks lightly but to argue this point paying due respect to his inspiring writings requires another separate paper. 
${ }^{\mathrm{c}}$ Some register families have a strong tendency to suppress dialectal/idiolectal characteristics; writing a lab report or applying for research funding are two such examples; in speaking, a job interview discourages prominent idiolectal features.

${ }^{\mathrm{d}}$ For more information see the Collected Works of M A K Halliday, Volumes 2: On Language and Linguistics, 2002b and Volume 10: Language and Society, 2007a. London: Continuum.

${ }^{\mathrm{e}}$ Hasan 1973 was first presented at a seminar at London School of Economics in 1969. It criticized the neglect of semantics, and offered reasons for preferring that stratum as the focus for the identification of the linguistic correlates.

${ }^{\mathrm{f}}$ These relations are evident in the last resort as the interdependence between certain classes of words in a clause. For example, compare the a-normal I invited him but he came with the normal I invited him but he didn't come.

${ }^{\mathrm{g}}$ Not all departures are necessarily random; in fact they are often 'conditioned' (Hasan 2011); one might for example consider field and transitivity in connection with the use of grammatical metaphors in scientific domain. Permeability might be an issue.

hI believe the use of the expression 'context metafunction hook up hypothesis' does injustice to Halliday's statements about the resonance of the metafunctions with contextual parameters. It misapplies the term 'hook up' which had been used by Halliday only to refer to the 'unifying' function of grammar as 'a device for hooking up together' into 'one unified structural form' the various elements derived from the metafunctional analysis (e.g., 1973: 42). Applying this term to context metafunction relations is to rely on a mistaken analogy.

'The earliest label had been 'general/ized formula' (Hasan 1978); later I also used the term 'generic' meaning thereby 'pertaining to species/type'. And even when Bakhtin's 'speech genre' achieved popularity, they tended to be associated with 'schematic structures'. Unlike the GSP, schematic structures were 'individuals', which suited the 'dynamic perspective' since its concern was not with type but with the process of each text. The schematic structures were not explicitly related to features of the $\mathrm{CC}$. The Generalized Formula had meanwhile wavered between Generalized Structure Potential and Generic Structure Potential.

${ }^{j}$ This is not to say that accidental moves whether material or semantic are 'meaningless'; however precisely because they are unpredictable, establishing a routine is not possible.

'I have long felt the need to explore the differences between 'act', 'action' and 'activity': this would bring greater order in understanding field as the parameter concerned with 'doing' of some kind.

${ }^{\mathrm{I}} \mathrm{I}$ am indebted to Margaret Berry (private conversation) for drawing attention to the distinction between option and choice. I take the responsibility for the extension and interpretation of the terms as presented here.

${ }^{\mathrm{m}}$ See also Butt (2013) for a revised system network of mode of discourse. The options written in capital letters e.g. 'ANCILLARY' or 'CONSTITUTIVE' in Butt (2004) kindly acknowledge these option names were used in my work that in pre-systemic discussions of the three parameters. I have not replicated this practice here: the terms, having been embedded in a largely new systemic environment, no longer mean the same thing. I will comment on this 'integration' of terms from distinct systems in section Concluding remarks: the value of a scientific study of con/text. 
${ }^{\mathrm{n}}$ Sometimes reference needs to be made to just a portion of the SE: I will refer to such a portion as a 'choice path'.

${ }^{\circ}$ These comments are based on Figures 6 (mode), Figure 7 (tenor) in this paper, whereas for field see Hasan, 1999, a revised version of which is presented in Hasan (in press).

${ }^{\mathrm{P}}$ Since each simple system represents a particular perspective, in principle, each should be assigned a name but for obvious reasons the inclusion of all the system names, instead of reducing uncertainty, will create confusion. It is, however, necessary for each simple system to have an address for easy reference; in my work I have tried to identify them by a combination of letters and numbers (Hasan, 2009b; 2013); Butt (2004) employs a decimal numbering system that within limits can be used for tracking delicacy.

'The term 'context of culture and situation' is sometimes abbreviated to just 'context' or just 'situation', neither of which by itself is a satisfactory focus, though it is a great space-saver! I have used the term 'context', except where the discourse requires clarification as for example in showing the difference between the 'context' and 'material situational setting' (Hasan, 1999; 2009a; in press a).

'I first called the arrow diacritic 'default dependency' (Hasan, 1999: 278), but, on reflection, what the arrow diacritic does is to pre-empt free choice, forcing the selection of one single 'default' choice; so, in fact, it is a form of pre-selection of options, except that the term 'pre-select' has been dedicated to another relation.

${ }^{\mathrm{s}}$ These were not built into a system but described discursively since the readership to which the writing was addressed could not be expected to have a working knowledge of sys-nets.

\section{Additional files}

Additional file 1: Appendix A: Glossary of options in Figure 6.

Additional file 2: Appendix B: SEs for systemic paths $C$ and $P$ in Figure 6.

Additional file 3: Appendix C: Glossary of options in Tenor Figure 7.

Competing interests

The author declares that she has no competing interests.

Received: 29 April 2014 Accepted: 7 August 2014

Published online: 28 November 2014

\section{References}

Abercrombie, David. 1965. R. P. and local accent. In Studies in Phonetics and Linguistics. London: Oxford University Press. First presented as R. P. and local accent in B. B. C. Broadcast, Third Programme, August 29, 1951.

Bernstein, Basil. 1990. The Structuring of Pedagogic Discourse: Class, Codes and Control, vol. 4. London: Routledge.

Bourdieu, Pierre. 1991. Introduction: the economy of linguistic exchange. In Language and Symbolic Power, ed. John B Thompson, Raymond $\operatorname{Tr}$ Gino, and Adamson Matthew. London: Polity Press.

Bowcher Wendy, L. 2007. Field and multimodal texts. In Continuing Discourse on Language: A Functional Perspective, ed. Hasan Ruqaiya, Matthiessen Christian, and Jonathan J Webster. London: Equinox.

Bowcher, Wendy L. 2014. Issues in developing unified systems for contextual field and mode. To appear in Functions of Language 12:2.

Brazelton, TB, B Koslowski, and M Main. 1974. The origin of reciprocity: the early mother-infant interaction. In The Effect of the Infant on its Caregiver, ed. M Lewis and LA Rosenblum. New York: Wiley.

Butt David, G. 2001. Firth, Halliday and the development of the systemic functional theory. In History of the Language Sciences: An International Handbook of the Evolution of the Study of Language from the Beginning to the Present ed. Auroux Sylvain, EFK Koerner, Niederehe Hans-Josef, and Versteegh Kees. Berlin: Walter de Gruyter.

Butt David, G. 2004. Parameters of Context: On Establishing Similarities and Dissimilarities between Social Processes. (Mimeo.). Sydney: Centre for Language in Social Life, Macquarie University. 
Butt David, G. 2005. Method and imagination in Halliday's science of linguistics. In Continuing Discourse on Language: A Functional Perspective, vol. 1, ed. Hasan Ruqaiya, Matthiessen Christian, and Jonathan J Webster. London: Equinox.

Butt, DG. 2013. The medium, the channel and the message: technologies of mind and matter in the current meaning of mode. A keynote presentation at Third Register and Context Symposium: Mode, Text and Texture. Macquarie University, Department of Linguistics. February 13-15, 2013. Video available at http:/Nimeo.com/62212145 (Part I) and http:/Nimeo.com/62212460 (Part 2).

Cloran, C. 1994. Rhetorical Units and Decontextualization: an Enquiry into Some Relations of Context, Meaning and Grammar. Monographs in Systemic Functional Linguistics, No. 6. Department of English Studies: Nottingham University.

Cloran, C. 1995. Defining and relating text segments: subject and theme in discourse. In On Subject and Theme: A Discourse Functional Perspective, ed. Hasan Ruqaiya and Peter H Fries. Amsterdam: John Benjamins.

Cloran, C. 1999. Context, material situation and text. In Text and Context in Functional Linguistics, ed. M Ghadessy. Amsterdam: John Benjamins.

Cloran, C. 2000. Socio-semantic variation: different wordings, different meanings. In Researching Language in School and Communities: Functional Linguistic Perspectives, ed. Unsworth Len. London: Cassell.

Davies, Eirian. 2014. A retrospective view of systemic functional linguistics with notes from a parallel perspective. Functional Linguistics 1.1.

de Saussure, Ferdinand. 2006. Writings in General Linguistics, ed. Bouquet Simon, Engler Rudolf, Weil Antoinette, Tr English, Sanders Carol, and Pires Matthew. Oxford: Oxford University Press.

de Saussure, Ferdinand. 1966. Course in General Linguistics, Tr. and Intro., Wade Baskin. New York: McGraw-Hill.

Fawcett, RP. 1980. Cognitive Linguistics and Social Interaction: Towards an Integrated Model of a Systemic Functional Grammar and Other Components of an Interactional Mind. Heidelberg: Julius Gross.

Fawcett, RP. 2000. A Theory of Syntax for Systemic Functional Linguistics. Amsterdam: Benjamins.

Firth, JR. 1957. Papers in Linguistics 1934-1951. London: Oxford University Press.

Firth, JR. 1968. Selected Papers of J R Firth, ed. Longmans: F R Palmer. London.

Fontaine, Lise, Tom Bartlett, and Gerard O'Grady. 2013. Systemic Functional Linguistics: Exploring Choice. Cambridge: Cambridge University Press.

Goffman, Erving. 1964. The neglected situation. In The Ethnography of Communication: American Anthropologist 66, 6, part II: 133-137, ed. John J Gumperz and Hymes Dell.

Gregory, Michael. 1967. Aspects of varieties differentiation. Journal of Linguistics 3:177-198.

Gregory, Michael, and Susanne Carroll. 1978. Language and Situation: Language Varieties and their Social Contexts. London: Routledge and Kegan Paul.

Halliday, MAK. 1961. Categories of the theory of grammar. Word 17(3):241-92.

Halliday, MAK. 1967-68. Notes on transitivity and theme in English. Journal of Linguistics . Part 1, JL 3.1: 37-81; Part 2, JL 3.2: 199-244; Part 3, JL 4.2:179-215.

Halliday, MAK. 1969. Options and functions in the English clause. Brno Studies in English 8:81-88.

Halliday, MAK. 1970a. Language structure and language function. In New Horizons in Linguistics, ed. Lyons John. England: Penguin: Harmondsworth.

Halliday, MAK. 1970b. Functional diversity in language, as seen from a consideration of modality and mood in English. Foundations of Language 6:322-361.

Halliday, MAK. 1973. Explorations in the functions of language. London: Arnold.

Halliday, MAK. 1975. Learning How to Mean: Explorations in Language development. London: Arnold.

Halliday, MAK. 1976. System and Function in Language: Selected Papers, ed. Gunther R Kress. London: Oxford University Press.

Halliday, MAK. 1979. Modes of meaning and modes of expression: types of grammatical structure and their determination by different semantic functions. In Function and Context in Linguistic Analysis. Essays in Honour of W. A. Hass, ed. DJ Allen, E Carney, and D Holdcroft. Cambridge: Cambridge University Press.

Halliday, MAK. 1984. An Introduction to Functional Grammar. London: Arnold.

Halliday, MAK. 1991. The Notion of 'Context' in language education. In Language Education: Interaction and Development: Proceedings of the International Conference, Vietnam 30 March - 1 April 1991, ed. Lê Thao and McCausland Mike. Launceston: University of Tasmania.

Halliday, MAK. 1994. An Introduction to Functional Grammar, 2nd ed. London: Edward Arnold.

Halliday, MAK. 2009. Methods - techniques - problems. In Continuum Companion to Systemic Functional Linguistics, ed. MAK Halliday and J Jonathan. London: Continuum: Webster.

Halliday, MAK, and Ruqaiya Hasan. 1976. Cohesion in English. London: Longman.

Halliday, MAK, and Ruqaiya Hasan. 1985. Language, Context and Text: Aspects of Language in a Social-Semiotic Perspective. Geelong, Vic:: Deakin University Press.

Halliday, MAK, Angus McIntosh, and Peter Strevens. 1964. Linguistic Sciences and Language Teaching. London: Longmans.

Halliday, MAK, MIM Christian, and Matthiessen. 1999. Construing Experience Through Meaning: A Language-based Approach to Cognition, 1999. London: Continuum.

Halliday, MAK, MIM Christian, and Matthiessen. 2014. Halliday's Introduction to Functional Grammar. $4^{\text {th }}$ Revision. London: Routledge.

Hasan, Ruqaiya. 1973. Code, register and social dialect. In Class, Codes and Control, Volume 2: Applied Studies towards a Sociology of Language, ed. B Bernstein. London: Routledge \& Kegan Paul.

Hasan, Ruqaiya. 1978. Text in the systemic-functional model. In Current Trends in Text-Linguistics, ed. Wolfgang U Dressler. Berlin: Mouton de Gruyter.

Hasan, Ruqaiya. 1983. A Semantic Network for the Analysis of Messages in Everyday Talk between Mothers and their children. (mimeo). North Ryde NSW: Macquarie University.

Hasan, Ruqaiya. 1985. The identity of a text. In Chapter 6: Part B of Language, Context, and Text: Aspects of Language in a Social Semiotic Perspective, ed. MAK Halliday and Hasan Ruqaiya. Geelong, Vic: Deakin University Press.

Hasan, Ruqaiya. 1987. The grammarian's dream: lexis as the most delicate grammar. In New Developments in Systemic Linguistics, Volume 1: Theory and Description, ed. MAK Halliday and Robin P Fawcett. London: Pinter. 
Hasan, Ruqaiya. 1995. The conception of context in text. In Discourse in Society: Systemic Functional Perspectives, ed. Peter H Fries and Gregory Michael. Norwood, NJ: Ablex.

Hasan, Ruqaiya. 1999. Speaking with reference to context. In Text and Context in Functional Linguistics, ed. Ghadessy Mohsen. Amsterdam: Benjamins.

Hasan, Ruqaiya. 2000. The uses of talk. In Discourse and Social Life, eds., Srikant Sarangi and Malcolm Coulthard. London: Pearson Education Ltd.

Hasan, Ruqaiya. 2001. Wherefore Context? The place of context in the system and process of language. In Grammar and Discourse: Proceedings of the International Conference on Discourse Analysis, ed. Shaozeng Ren, Guthrie William, and IW Ronald Fong. Macau: University of Macau.

Hasan, Ruqaiya. 2009a. The place of context in a systemic functional model. In Continuum Companion to Systemic Functional Linguistics, ed. MAK Halliday and Jonathan J Webster. London: Continuum.

Hasan, Ruqaiya. 2009b. Collected Works of Ruqaiya Hasan, Volume 2. In Semantic Variation: Meaning in Society and in Sociolinguistics, ed. Jonathan J Webster. London: Equinox.

Hasan, Ruqaiya. 2011. Collected Works of Ruqaiya Hasan, Volume 3: Language and Education: Learning and Teaching in Society, ed. Equinox: Jonathan J Webster. London.

Hasan, Ruqaiya. 2013. Choice, System, Realisation: Describing Language as Meaning Potential. In Systemic Functional Linguistics: Exploring Choice, ed. Fontaine Lise, Bartlett Tom, and O'Grady Gerald. Cambridge: Cambridge University Press.

Hasan, Ruqaiya. 2014. Linguistic sign and the science of linguistics: the foundations of appliability. In Developing Systemic Functional Linguistics, ed. Yan Fang and Jonathan J Webster. London: Equinox.

Hasan, Ruqaiya. in press aa. Tenor: rethinking interactant relations. In The Collected Works of Ruqaiya Hasan, Volume 4: Context in the System and Process of Language, ed. Jonathan J Webster. London: Equinox.

Hasan, Ruqaiya. In press bb. In the nature of language: reflections on permeability and hybridity. In Hybridity in Systemic Functional Linguistics: Grammar, Text and Discursive Context, ed. Donna R Miller and Bayley Paul. London 2015: Equinox.

Hjelmslev, Louis. 1961. Prolegomena to a Theory of Language. (Translated \& edited by F. J. Whitfield). Madison: University of Wisconsin Press.

Hymes, Dell. 1986. Models of the interaction of language and social life. In Directions in Sociolinguistics: the Ethnography of Communication, ed. John J Gumperz and Hymes Dell. Oxford: Basil Blackwell. revised edition.

Kress, Gunther. 1976. English system Networks. In Halliday: system and Function in Language: Selected Papers, ed., Gunther Kress. London: Oxford University Press.

Lamb Sydney, M, and Leonard E Newell. 1966. Outline of Stratificational Grammar: with an appendix. Washington, D.C: Georgetown University Press.

Lock, A. 1978. Action, Gesture and Symbol: the Emergence of Language. New York: Academic Books.

Malinowski, Bronislaw. 1923. The problem of meaning in primitive languages. Supplement I to C. K. Ogden and I. A Richards, The Meaning of Meaning. New York, NY: Harcourt Brace and World.

Malinowski, Bronislaw. 1935. An ethnographic theory of language, and some corollaries. In Coral Gardens and Their Magic: the language of magic and gardening, Volume Two Part IV. London: Allen and Unwin.

Mann, William. 1985. An introduction to the Nigel text generation grammar. In Systemic Perspectives on Discourse, Volume 1, ed. James Benson and William S Greaves. Norwood, NJ: Ablex.

Martin, James R. 1992. English Text: System and Structure. Amsterdam: Benjamins.

Martin, James R. 2013. Systemic Functional Grammar: A Next Step into the Theory: Axial Relations. Beijing: Higher Education Press.

Matthiessen Christian, MIM. 1995. Lexicogrammatical Cartography: English Systems. Tokyo: International Language Science Publishers.

Matthiessen Christian, MIM. 2007. The architecture of language according to systemic functional theory: developments since the 1970s. In Continuing Discourse on Language: Functional Perspectives, vol. 2, ed. Hasan Ruqaiya, Matthiessen Christian, and Jonathan J Webster. London: Equinox

Matthiessen Christian, MIM. 2009. Ideas and new directions. In Continuum Companion to Systemic Functional Linguistics, ed. MAK Halliday and Jonathan J Webster. London: Continuum.

Matthiessen Christian, MIM. in press. Halliday on language. In Companion to Halliday, ed. Jonathan J Webster. London: Bloomsbury.

Trevarthen, Colwyn. 1974. Conversations with a two month old. New Scientist 62:230-235.

Ure, Jean, and Jeff Ellis. 1977. Register in descriptive linguistics and in linguistic sociology. In Issues in Sociolinguistics, ed. O Uribe-Villas. The Hague: Mouton.

Vygotsky Lev, Semyonovich. 1978. Mind in Society: The development of Higher Psychological Processes, ed. Cole Michael, John-Steiner Vera, and Souberman Ellen. Cambridge, MA: Harvard University Press.

Williams, Geoff. 1995. Joint Book-Reading and Literacy Pedagogy: a Socio-Semantic Interpretation. Doctoral dissertation. Sydney: Department of Linguistics, Macquarie Univer.

doi:10.1186/s40554-014-0009-y

Cite this article as: Hasan: Towards a paradigmatic description of context: systems, metafunctions, and semantics. Functional Linguistics 2014 1:9. 